DOI 10.4171/JEMS/384

Qun Chen · Jürgen Jost · Guofang Wang · Miaomiao Zhu

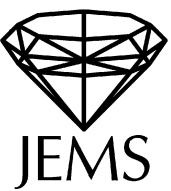

\title{
The boundary value problem for Dirac-harmonic maps
}

Received October 12, 2010 and in revised form June 17, 2011

\begin{abstract}
Dirac-harmonic maps are a mathematical version (with commuting variables only) of the solutions of the field equations of the non-linear supersymmetric sigma model of quantum field theory. We explain this structure, including the appropriate boundary conditions, in a geometric framework. The main results of our paper are concerned with the analytic regularity theory of such Dirac-harmonic maps. We study Dirac-harmonic maps from a Riemannian surface to an arbitrary compact Riemannian manifold. We show that a weakly Dirac-harmonic map is smooth in the interior of the domain. We also prove regularity results for Dirac-harmonic maps at the boundary when they solve an appropriate boundary value problem which is the mathematical interpretation of the D-branes of superstring theory.
\end{abstract}

Keywords. Dirac-harmonic map, regularity, boundary value

\section{Introduction}

In [6], a variational problem has been introduced that is an analogue for ordinary, that is, commuting fields of the non-linear supersymmetric sigma model of quantum field theory. Of course, this model is no longer supersymmetric, but it does share the other symmetries of the sigma model, in particular conformal invariance. Also, this model has a surprisingly subtle geometric and analytic structure. In the present paper, we explore some further geometric and analytic aspects. In particular, we look at boundary conditions that are of the type of the D-branes of superstring theory and involve the chirality operator of a spin structure. After a careful geometric derivation of these boundary conditions, we shall provide the analytic regularity theory for solutions of the field equations at such a boundary.

Q. Chen: School of Mathematics and Statistics, Wuhan University, Wuhan 430072, China; e-mail: qunchen@whu.edu.cn

J. Jost: Max Planck Institute for Mathematics in the Sciences, Inselstr. 22, D-04103 Leipzig, Germany, and

Department of Mathematics, University of Leipzig, D-04081 Leipzig, Germany; e-mail: jost@mis.mpg.de

G. Wang: Mathematisches Institut, Albert-Ludwigs-Universität Freiburg,

D-79104 Freiburg, Germany; e-mail: Guofang.Wang@math.uni-freiburg.de

M. Zhu: Department of Mathematics, ETH Zürich, CH-8092, Zürich, Switzerland; present address: Mathematics Institute, University of Warwick, CV4 7AL, Coventry, UK; e-mail: Miaomiao.Zhu@warwick.ac.uk 
Let us now describe the model in some more detail. For the non-linear supersymmetric sigma model of quantum field theory (see e.g. [8] or [21] for mathematical background), one considers a map

$$
Y: M^{s} \rightarrow N
$$

from a (2|2)-dimensional supermanifold $M^{s}$ to some Riemannian manifold $N$. With local even coordinates $x^{1}, x^{2}$ and odd (i.e., anticommuting) coordinates $\theta^{1}, \theta^{2}$, the action is

$$
S=\int \frac{1}{4} \epsilon^{\alpha \beta}\left\langle D_{\alpha} Y, D_{\beta} Y\right\rangle d d^{2} x d d \theta^{2} d d \theta^{1}
$$

where $\epsilon$ is the usual antisymmetric tensor, the brackets $\langle\cdot, \cdot\rangle$ denote the Riemannian metric on $N$ (by conformal invariance, we may assume that the domain metric is flat), and $d \theta$ indicates that a Berezin integral has to be taken.

The map $Y$ has the following expansion:

$$
Y=\phi(x)+\psi_{\alpha}(x) \theta^{\alpha}+F(x) \theta^{1} \theta^{2} .
$$

Here, $\phi$ is an ordinary map from the ordinary manifold $M$ underlying the supermanifold $M^{s}$ into $N$; in fact, $M$, being 2-dimensional, is considered as a Riemann surface. Moreover $\psi$ is an anticommuting spinor with values in the pull-back tangent bundle $\phi^{-1} T N$. In fact, $\psi$ is a real Euclidean Majorana spinor with respect to a real 2-dimensional Euclidean representation of the Clifford algebra $C l(2,0)$. The field $F$ is needed to close the supersymmetry algebra off-shell, but will not be of importance for our subsequent purposes.

Using this expansion and carrying out the $\theta$-integration, the Lagrangian density in (1.2) becomes

$$
\frac{1}{2}\|d \phi\|^{2}+\frac{1}{2}\langle\psi, \not D \psi\rangle-\frac{1}{12} \epsilon^{\alpha \beta} \epsilon^{\gamma \delta}\left\langle\psi_{\alpha}, R\left(\psi_{\beta}, \psi_{\gamma}\right) \psi_{\delta}\right\rangle .
$$

$\not D$ is the Dirac operator along the map $\phi$; it involves the ordinary Dirac operator $\not \partial$ of $M$ and the Levi-Civita connection of $N$ (see e.g. $[6,21]$ ). $\|\cdot\|$ indicates again the metric of $N$, and $R$ is its curvature. In fact, the curvature term arises from the Berezin integration of the $F$-term, and again, we shall not need it below.

The reason why the spinor field $\psi$ is taken as odd is that for $\psi$ even, $\langle\psi, \not D \psi\rangle$ would vanish upon integration by parts. This in turn results from the fact that we are working with a Clifford algebra $(C l(2,0)$ in the present case) with a real representation. Were the representation imaginary, in contrast, the integral of $\langle\psi, \not D \psi\rangle$ would vanish for $\psi$ odd, but no longer for $\psi$ even. Of course, $C l(2,0)$ does not have such a representation, but the Clifford algebra $C l(0,2)$ does. This is the basis of the model of [6].

To be concrete, consider the representation of $C l(0,2)$ with

$$
e_{1} \mapsto \gamma_{1}=\left(\begin{array}{cc}
0 & i \\
i & 0
\end{array}\right), \quad e_{2} \mapsto \gamma_{2}=\left(\begin{array}{cc}
0 & 1 \\
-1 & 0
\end{array}\right)
$$

acting on spinors. For a spinor field $\omega: \mathbb{R}^{2} \rightarrow \mathbb{C}^{2}$, we then have the Dirac operator

$$
\not \partial=\left(\begin{array}{ll}
0 & i \\
i & 0
\end{array}\right)\left(\begin{array}{l}
\partial \omega_{1} / \partial x_{1} \\
\partial \omega_{2} / \partial x_{1}
\end{array}\right)+\left(\begin{array}{cc}
0 & 1 \\
-1 & 0
\end{array}\right)\left(\begin{array}{l}
\partial \omega_{1} / \partial x_{2} \\
\partial \omega_{2} / \partial x_{2}
\end{array}\right)=2 i\left(\begin{array}{l}
\partial \omega_{2} / \partial z \\
\partial \omega_{1} / \partial \bar{z}
\end{array}\right),
$$


that is, the Cauchy-Riemann operator. Let $\omega$ and $\psi$ be two spinor fields with compact support on $\mathbb{R}^{2}$; we then have the integration by parts formula

$$
\int\langle\omega, \not \partial \psi\rangle=\int\langle\not \omega, \psi\rangle
$$

that is, $\not \partial$ is formally self-adjoint.

We can thus introduce the model of [6]. Let $M$ be a Riemann spin surface, $\Sigma M$ the spinor bundle over $M$, and $N$ a compact Riemannian manifold without boundary. Let $\phi$ be a map from $M$ to $N$, and $\psi$ a section of the bundle $\Sigma M \otimes \phi^{-1} T N$. Let $\widetilde{\nabla}$ be the connection induced from those on $\Sigma M$ and $\phi^{-1} T N$. The Dirac operator $\not D$ along the map $\phi$ is defined by $\not D \psi:=\gamma_{\alpha} \cdot \widetilde{\nabla}_{\gamma_{\alpha}} \psi$, where $\gamma_{\alpha}$ is a local orthonormal frame on $M$. We consider the functional

$$
L(\phi, \psi):=\int_{M}\left(\|d \phi\|^{2}+\langle\psi, \not D \psi\rangle\right)
$$

Except for the curvature term (which we do not need as we are not concerned with supersymmetry), the Lagrangian density here is formally the same as in (1.4). However, in (1.8), all fields are commuting.

The critical points $(\phi, \psi)$ of (1.8) are called Dirac-harmonic maps from $M$ to $N$. They constitute the object of our study in this paper.

The focus of our paper is on boundary conditions and boundary regularity for such maps. The first issue is the identification of the correct boundary conditions. In a certain sense, we are translating the boundary conditions of the non-linear supersymmetric sigma model (see [1, 2]), into a geometric framework. Our Riemannian geometric perspective will clarify some geometric aspects. Let thus $M$ be a Riemann surface with boundary $\partial M$. This boundary should be mapped to a D-brane. Geometrically, this means that we have a submanifold $\mathcal{S}$ of $N$, and $\phi(\partial M)$ should be contained in $\mathcal{S}$ in such a way that it is critical for (1.8) with respect to to all such boundary values. This simply means that, in the absence of the field $\psi, \phi(\partial M)$ should meet $\mathcal{S}$ orthogonally. In the harmonic map literature, this is called a free boundary condition with support $\mathcal{S}$. In analytic terms, this is a combination of Dirichlet and Neumann boundary conditions. Analytically, this is usually treated by some reflection method (see e.g. [13, 20, 26]). That is, one doubles $M$ to $\bar{M}$ by reflection across the boundary $\partial M$ and extends $\phi$ to $\bar{M}$ by reflection across the submanifold $\mathcal{S}$. This clarifies the geometric meaning of the tensor $R$ utilized in [1, 2], as we shall explain in more detail below. In any case, the reflection across $\mathcal{S}$ is particularly well controlled when $\mathcal{S}$ is a totally geodesic submanifold of $N$. This condition is also required (in different terminology) in $[1,2]$. In fact, we shall not need this condition for the formulation of the boundary condition, nor for the proof of continuity of our solutions, but we shall need it to get higher regularity of solutions at the boundary.

As our model couples the harmonic map equation to a Dirac type equation, besides the regularity theory for harmonic maps, also the one for solutions of Dirac equations, in the interior and at the boundary, is relevant. Some pertinent references are $[3,4,5,9$, 23]. In our setting, for the spinor $\psi$ we shall need a chirality boundary condition (first introduced by Gibbons-Hawking-Horowitz-Perry [10]). We explain this here only for the 
linear case. The coupling between the boundary conditions for the fields $\phi$ and $\psi$ in the non-linear case will be worked out in detail below. Mathematically, the chirality condition is explained in [16]. We consider the chirality operator $G=i \gamma_{1} \gamma_{2}$, and we can decompose the spinor bundle $\Sigma M$ of $M$ into the eigensubbundles of $G$ for the eigenvalues \pm 1 . Restricting to the boundary, we have the decomposition $\mathbf{S}:=\left.\Sigma M\right|_{\partial M}=V^{+} \oplus V^{-}$. With $\vec{n}$ being the outward unit normal vector field on $\partial M$, the orthogonal projection onto the eigensubbundle $V^{ \pm}$

$$
\mathbf{B}^{ \pm}: L^{2}(\mathbf{S}) \rightarrow L^{2}\left(V^{ \pm}\right), \quad \psi \mapsto \frac{1}{2}(I \pm \vec{n} G) \psi,
$$

defines a local elliptic boundary condition for the Dirac operator $\not \partial$ (see [16]). We say a spinor $\psi \in W^{1,4 / 3}(\Sigma M)$ satisfies the boundary condition $\mathbf{B}^{ \pm}$if

$$
\left.\mathbf{B}^{ \pm} \psi\right|_{\partial M}=0 .
$$

Our main analytical results are then concerned with weak solutions of the field equations with (1.8), that is, for weakly Dirac-harmonic maps (again, see the main text, e.g. Definition 2.1, for a precise definition) with such boundary conditions. We shall prove

Theorem 1.1. Let $M$ be a compact Riemann spin surface with boundary $\partial M, N$ be any compact Riemannian manifold, and $\mathcal{S}$ be a closed submanifold of $N$. Let $(\phi, \psi)$ be a weakly Dirac-harmonic map from $M$ to $N$ with free boundary on $\mathcal{S}$. Then for any $\alpha \in$ $(0,1)$,

$$
\phi \in C^{0, \alpha}(M, N)
$$

Theorem 1.2. Let $M$ be a compact Riemann spin surface with boundary $\partial M, N$ be any compact Riemannian manifold, and $\mathcal{S}$ be a closed, totally geodesic submanifold of $N$. Let $(\phi, \psi)$ be a weakly Dirac-harmonic map from $M$ to $N$ with free boundary on $\mathcal{S}$ and suppose that $\phi \in C^{0, \alpha}(M, N)$ for any $\alpha \in(0,1)$. Then there exists $\beta \in(0,1)$ such that

$$
\phi \in C^{1, \beta}(M, N), \quad \psi \in C^{1, \beta}\left(\Sigma M \otimes \phi^{-1} T N\right) .
$$

In fact, we shall start by showing the regularity of weakly Dirac-harmonic maps in the interior of $M$. This was shown independently by Wang-Xu [28] by a different method inspired by [24, 25]. Our methods will also utilize the general strategy of Rivière [24] who had achieved an important generalization of the earlier results of Wente [27] and Hélein $[14,15]$. Rivière's approach has been adapted to Dirichlet boundary regularity by Müller-Schikorra [22], and this work will also be useful for our purposes.

\section{Interior regularity}

Let $M$ be a Riemann surface equipped with a conformal metric, which by conformal invariance of our functionals can then be assumed to be Euclidean, and with a fixed spin structure, $\Sigma M$ the spinor bundle, and let $\phi$ be a smooth map from $M$ to another Riemannian manifold $(N, g)$ of dimension $d \geq 2$. Denote by $\phi^{-1} T N$ the pull-back bundle of $T N$ 
by $\phi$ and consider the twisted bundle $\Sigma M \otimes \phi^{-1} T N$. On $\Sigma M \otimes \phi^{-1} T N$ there is a metric induced from the metrics on $\Sigma M$ and $\phi^{-1} T N$. Also we have a natural connection $\widetilde{\nabla}$ on $\Sigma M \otimes \phi^{-1} T N$ induced from those on $\Sigma M$ and $\phi^{-1} T N$. In local coordinates, the section $\psi$ of $\Sigma M \otimes \phi^{-1} T N$ can be expressed as

$$
\psi(x)=\sum_{j=1}^{d} \psi^{j}(x) \otimes \partial y^{j}(\phi(x)),
$$

where $\psi^{j}$ is a spinor and $\left\{\partial y^{j}\right\}$ is the natural local basis on $N$. Then $\widetilde{\nabla}$ can be expressed as

$$
\widetilde{\nabla} \psi=\sum_{i=1}^{d} \nabla \psi^{i}(x) \otimes \partial y^{i}(\phi(x))+\sum_{i, j, k=1}^{d} \Gamma_{j k}^{i}(\phi(x)) \nabla \phi^{j}(x) \cdot \psi^{k}(x) \otimes \partial y^{i}(\phi(x)) .
$$

Now we define the Dirac operator along the map $\phi$ by

$$
\begin{aligned}
\not D \psi & :=\gamma_{\alpha} \cdot \widetilde{\nabla}_{\gamma_{\alpha}} \psi \\
& =\sum_{i} \not \partial \psi^{i}(x) \otimes \partial y^{i}(\phi(x))+\sum_{i, j, k=1}^{d} \Gamma_{j k}^{i}(\phi(x)) \nabla_{\gamma_{\alpha}} \phi^{j}(x) \gamma_{\alpha} \cdot \psi^{k}(x) \otimes \partial y^{i}(\phi(x)),
\end{aligned}
$$

where $\gamma_{1}, \gamma_{2}$ is the local orthonormal frame on $M$ and $\not \partial:=\sum_{\alpha=1}^{2} \gamma_{\alpha} \cdot \nabla_{\gamma_{\alpha}}$ is the usual Dirac operator.

Set

$$
\mathcal{X}(M, N):=\left\{(\phi, \psi) \mid \phi \in C^{\infty}(M, N) \text { and } \psi \in C^{\infty}\left(\Sigma M \otimes \phi^{-1} T N\right)\right\} .
$$

On $\mathcal{X}(M, N)$, we consider the following functional

$$
L(\phi, \psi):=\int_{M}\left[|d \phi|^{2}+(\psi, \not D \psi)\right]=\int_{M}\left[g_{i j}(\phi) \frac{\partial \phi^{i}}{\partial x_{\alpha}} \frac{\partial \phi^{j}}{\partial x_{\alpha}}+g_{i j}(\phi)\left(\psi^{i}, \not D \psi^{j}\right)\right] .
$$

(Recall that the domain metric can be taken to be Euclidean.) The Euler-Lagrange equations of $L(\cdot, \cdot)$ are

$$
\begin{aligned}
& \tau^{m}(\phi)-\frac{1}{2} R_{l i j}^{m}(\phi)\left(\psi^{i}, \nabla \phi^{l} \cdot \psi^{j}\right)=0, \quad m=1, \ldots, d, \\
& \not D \psi^{i}=\not \partial \psi^{i}+\Gamma_{j k}^{i}(\phi) \partial_{\alpha} \phi^{j} \gamma_{\alpha} \cdot \psi^{k}=0, \quad i=1, \ldots, d,
\end{aligned}
$$

where $\tau(\phi)$ is the tension field of the map $\phi$. Solutions $(\phi, \psi)$ to (2.10) and (2.11) are called Dirac-harmonic maps from $M$ to $N$.

Let $\left(N^{\prime}, g^{\prime}\right)$ be another Riemannian manifold and $f: N \rightarrow N^{\prime}$ a smooth map. For any $(\phi, \psi) \in \mathcal{X}(M, N)$ we set

$$
\phi^{\prime}=f \circ \phi \quad \text { and } \quad \psi^{\prime}=f_{*} \psi
$$


It is clear that $\psi^{\prime}$ is a spinor along the map $\phi^{\prime}$. Let $A$ be the second fundamental form of $f$, i.e., $A(X, Y)=\left(\nabla_{X} d f\right)(Y)$ for any $X, Y \in \Gamma(T N)$. The tension fields of $\phi$ and $\phi^{\prime}$ are related by

$$
\tau^{\prime}\left(\phi^{\prime}\right)=\sum_{\alpha=1}^{2} A\left(d \phi\left(\gamma_{\alpha}\right), d \phi\left(\gamma_{\alpha}\right)\right)+d f(\tau(\phi)) .
$$

It is also easy to check that the Dirac operators $\not D$ and $\not D^{\prime}$ corresponding to $\phi$ and $\phi^{\prime}$ respectively are related by

$$
\not D^{\prime} \psi^{\prime}=f_{*}(\not D \psi)+\mathcal{A}\left(d \phi\left(\gamma_{\alpha}\right), \gamma_{\alpha} \cdot \psi\right),
$$

where $\mathcal{A}\left(d \phi\left(\gamma_{\alpha}\right), \gamma_{\alpha} \cdot \psi\right):=\phi_{\alpha}^{i} \gamma_{\alpha} \cdot \psi^{j} \otimes A\left(\partial y^{i}, \partial y^{j}\right)$. Furthermore, if $f: N \rightarrow N^{\prime}$ is an isometric immersion, then $A(\cdot, \cdot)$ is the second fundamental form of the submanifold $N$ in $N^{\prime}$, and

$$
\nabla_{X}^{\prime} \xi=-P(\xi ; X)+\nabla_{X}^{\perp} \xi, \quad \nabla_{X}^{\prime} Y=\nabla_{X} Y+A(X, Y),
$$

for all $X, Y \in \Gamma(T N)$ and $\xi \in \Gamma\left(T^{\perp} N\right)$, where $P(\xi ; \cdot)$ denotes the shape operator. We can rewrite equations (2.10) and (2.11) in terms of $\mathcal{A}$ and the geometric data of the ambient space $N^{\prime}$.

Denote

$$
R(\phi, \psi):=\frac{1}{2} R_{l i j}^{m}\left(\psi^{i}, \nabla \phi^{l} \cdot \psi^{j}\right) \otimes \partial y^{m} .
$$

By the equation of Gauss, we have (see $[6,7,19,29]$ )

$$
R(\phi, \psi)=\operatorname{Re} \mathcal{P}\left(\mathcal{A}\left(d \phi\left(\gamma_{\alpha}\right), \gamma_{\alpha} \cdot \psi\right) ; \psi\right)+R^{\prime}(\phi, \psi),
$$

where $\mathcal{P}\left(\mathcal{A}\left(d \phi\left(\gamma_{\alpha}\right), \gamma_{\alpha} \cdot \psi\right) ; \psi\right):=P\left(A\left(\partial y^{l}, \partial y^{j}\right) ; \partial y^{i}\right)\left\langle\psi^{i}, \gamma_{\alpha} \cdot \psi^{j}\right\rangle \phi_{\alpha}^{l}$. Therefore, by using (2.12) and (2.13), and identifying $\phi$ with $\phi^{\prime}$ and $\psi$ with $\psi^{\prime}$, we can rewrite (2.10) and (2.11) as follows:

$$
\begin{aligned}
\tau^{\prime}(\phi) & =A\left(d \phi\left(\gamma_{\alpha}\right), d \phi\left(\gamma_{\alpha}\right)\right)+\operatorname{Re} \mathcal{P}\left(\mathcal{A}\left(d \phi\left(\gamma_{\alpha}\right), \gamma_{\alpha} \cdot \psi\right) ; \psi\right)+R^{\prime}(\phi, \psi), \\
\not D^{\prime} \psi & =\mathcal{A}\left(d \phi\left(\gamma_{\alpha}\right), \gamma_{\alpha} \cdot \psi\right) .
\end{aligned}
$$

In order to introduce the notion of weak solutions of the Euler-Lagrange equations, we embed $N$ isometrically into some $N^{\prime}=\mathbb{R}^{K}$ via the Nash-Moser embedding theorem. Then the above equations become

$$
\begin{aligned}
-\Delta \phi & =A(d \phi, d \phi)+\operatorname{Re} \mathcal{P}\left(\mathcal{A}\left(d \phi\left(\gamma_{\alpha}\right), \gamma_{\alpha} \cdot \psi\right) ; \psi\right), \\
\not \partial \psi & =\mathcal{A}\left(d \phi\left(\gamma_{\alpha}\right), \gamma_{\alpha} \cdot \psi\right) .
\end{aligned}
$$

Denote

$$
\begin{aligned}
H^{1}(M, N):= & \left\{\phi \in H^{1}\left(M, \mathbb{R}^{K}\right) \mid \phi(x) \in N \text { a.e. } x \in M\right\} \\
W^{1,4 / 3}(\Sigma M \otimes & \left.\phi^{-1} T N\right) \\
& :=\left\{\left.\psi \in \Gamma\left(\Sigma M \otimes \phi^{-1} T N\right)\left|\int_{M}\right| \nabla \psi\right|^{4 / 3}<\infty, \int_{M}|\psi|^{4}<\infty\right\} .
\end{aligned}
$$


Here, $\psi \in \Gamma\left(\Sigma M \otimes \phi^{-1} T N\right)$, the spinor field along the map $\phi$, should be understood as a $K$-tuple of spinors $\left(\psi^{1}, \ldots, \psi^{K}\right)$ satisfying

$$
\sum_{i} v_{i} \psi^{i}=0 \quad \text { for any normal vector } v=\sum_{i=1}^{K} v_{i} E_{i} \text { at } \phi(x),
$$

where $\left\{E_{i} \mid i=1, \ldots, K\right\}$ is the standard basis of $\mathbb{R}^{K}$. Denote

$$
\mathcal{X}_{1,4 / 3}^{1,2}(M, N):=\left\{(\phi, \psi) \in H^{1}(M, N) \times W^{1,4 / 3}\left(\Sigma M \otimes \phi^{-1} T N\right)\right\} .
$$

Critical points $(\phi, \psi) \in \mathcal{X}_{1,4 / 3}^{1,2}(M, N)$ of the functional $L(\cdot, \cdot)$ are called weakly Diracharmonic maps from $M$ to $N$ (see [7]); equivalently, we have

Definition 2.1. We call $(\phi, \psi) \in \mathcal{X}_{1,4 / 3}^{1,2}(M, N)$ a weakly Dirac-harmonic map from $M$ to $N$ if

$$
\begin{aligned}
& \int_{M}\left[\langle d \phi, d \eta\rangle+\left\langle A(d \phi, d \phi)+\operatorname{Re} \mathcal{P}\left(\mathcal{A}\left(d \phi\left(\gamma_{\alpha}\right), \gamma_{\alpha} \cdot \psi\right) ; \psi\right), \eta\right\rangle\right]=0, \\
& \int_{M}\left[\langle\psi, \not \partial \xi\rangle-\left\langle\mathcal{A}\left(d \phi\left(\gamma_{\alpha}\right), \gamma_{\alpha} \cdot \psi\right), \xi\right\rangle\right]=0
\end{aligned}
$$

for all $\eta \in H_{0}^{1} \cap L^{\infty}\left(M, \mathbb{R}^{K}\right)$ and $\xi \in W_{0}^{1,4 / 3} \cap L^{\infty}\left(\Sigma M \otimes \mathbb{R}^{K}\right)$.

Let us recall the following regularity result for two-dimensional conformally invariant variational problems by Rivière [24]. Denote by $B_{1}:=\left\{\left(x_{1}, x_{2}\right) \in \mathbb{R}^{2} \mid x_{1}^{2}+x_{2}^{2} \leq 1\right\}$ the unit disk in $\mathbb{R}^{2}$ and write $z=x_{1}+i x_{2}$.

Theorem A. Let $u \in H^{1}\left(B_{1}, \mathbb{R}^{K}\right)$ be a weak solution of

$$
-\Delta u=\Omega \cdot \nabla u
$$

where $\Omega=\left(\Omega_{j}^{i}\right)_{1 \leq i, j \leq K} \in L^{2}\left(B_{1}, \operatorname{so}(K) \otimes \mathbb{R}^{2}\right)$. Then $u$ is continuous.

To prove the smoothness of weakly Dirac-harmonic maps, it is sufficient to show the continuity of the map (see [7]):

Theorem B. Let $(\phi, \psi): B_{1} \rightarrow N$ be a weakly Dirac-harmonic map. If $\phi$ is continuous, then $(\phi, \psi)$ is smooth.

When $N=\mathbb{S}^{d}$, the continuity of weakly Dirac-harmonic maps was proved by Chen-JostLi-Wang [7], using Wente's Lemma [27]. Zhu [29] extended this result to the case that $N$ is a compact hypersurface in the Euclidean space $\mathbb{R}^{d+1}$. The case of a general target $N$ was shown independently by Wang-Xu [28], where Hélein's technique of moving frame $[14,15]$ and the Coulomb gauge construction, due to Rivière [24] and Rivière-Struwe [25], are combined.

Here, following the notations in [29], we show that the extrinsic equations (2.17) in the case of a general compact target can also be written in the same form as (2.21) and hence can be used to prove the continuity of weakly Dirac-harmonic maps. 
Theorem 2.1. Let $M$ be a Riemann spin surface, $N$ be any compact Riemannian manifold, and $(\phi, \psi)$ a weakly Dirac-harmonic map from $M$ to $N$. Then $\phi$ is continuous in the interior of $M$, and consequently $(\phi, \psi)$ is smooth.

Proof. We follow the approach in [29]. We assume without loss of generality that $M=B_{1}$ and take the orthonormal basis $\gamma_{1}=\partial_{x_{1}}, \gamma_{2}=\partial_{x_{2}}$. Fix a canonical coordinate $\left(y^{1}, \ldots, y^{K}\right)$ of $\mathbb{R}^{K}$. Let $v_{l}, l=d+1, \ldots, K$, be an orthonormal frame field for the normal bundle $T^{\perp} N$ to $N$ (the target $N$ considered is always assumed to be oriented). Denote by $v_{l}$ the corresponding unit normal vector field along the map $\phi$. We write

$$
\phi=\phi^{i} \partial y^{i}, \quad \psi=\psi^{j} \otimes \partial y^{j},
$$

and denote $\phi_{\alpha}:=\phi_{*}\left(\gamma_{\alpha}\right)=\phi_{x_{\alpha}}, \alpha=1,2$. Then, we proceed as in [29] to write (2.17) and (2.18) in the following extrinsic form in terms of the orthonormal frame field $v_{l}$, $l=d+1, \ldots, K$, for $T^{\perp} N$ :

$$
\begin{aligned}
-\Delta \phi^{m}= & \phi_{\alpha}^{i}\left(\phi_{\alpha}^{j} \frac{\partial v_{l}^{i}}{\partial y^{j}} v_{l}^{m}-\phi_{\alpha}^{j} \frac{\partial v_{l}^{m}}{\partial y^{j}} v_{l}^{i}\right) \\
& +\phi_{\alpha}^{i}\left\langle\psi^{k}, \gamma_{\alpha} \cdot \psi^{j}\right\rangle\left(\frac{\partial v_{l}^{i}}{\partial y^{j}}\left(\frac{\partial v_{l}}{\partial y^{k}}\right)^{\top, m}-\frac{\partial v_{l}^{i}}{\partial y^{k}}\left(\frac{\partial v_{l}}{\partial y^{j}}\right)^{\top, m}\right), \\
\not \partial \psi^{m}= & \frac{\partial v_{l}^{i}}{\partial y^{j}} v_{l}^{m} \phi_{\alpha}^{i} \gamma_{\alpha} \cdot \psi^{j} .
\end{aligned}
$$

Here $\top$ denotes the orthogonal projection $\mathbb{R}^{K} \rightarrow T_{y} N$ and $(\cdot)^{i}$ denotes the $i$-th component of a vector in $\mathbb{R}^{K}$. Note that $\phi_{\alpha} \in T N$ and $\left(\partial v_{l} / \partial y^{j}\right)^{\perp} \in T^{\perp} N$, hence

$$
\sum_{i} \phi_{\alpha}^{i}\left(\frac{\partial v_{l}}{\partial y^{j}}\right)^{\perp, i}=0, \quad \forall \alpha, l, j
$$

where $\perp$ denotes the orthogonal projection $\mathbb{R}^{K} \rightarrow T_{y}^{\perp} N$. Decomposing the vector $\partial v_{l} / \partial y^{j}$ into its tangent part and normal part and then applying (2.24), we get

$$
\frac{\partial v_{l}^{i}}{\partial y^{j}} \phi_{\alpha}^{i}=\left(\frac{\partial v_{l}}{\partial y^{j}}\right)^{i} \phi_{\alpha}^{i}=\left(\left(\frac{\partial v_{l}}{\partial y^{j}}\right)^{\top, i}+\left(\frac{\partial v_{l}}{\partial y^{j}}\right)^{\perp, i}\right) \phi_{\alpha}^{i}=\left(\frac{\partial v_{l}}{\partial y^{j}}\right)^{\top, i} \phi_{\alpha}^{i} .
$$

Thus, the equations (2.22) and (2.23) become

$$
\begin{aligned}
-\Delta \phi^{m}= & \phi_{\alpha}^{i}\left(\phi_{\alpha}^{j} \frac{\partial v_{l}^{i}}{\partial y^{j}} v_{l}^{m}-\phi_{\alpha}^{j} \frac{\partial v_{l}^{m}}{\partial y^{j}} v_{l}^{i}\right) \\
& +\phi_{\alpha}^{i}\left\langle\psi^{k}, \gamma_{\alpha} \cdot \psi^{j}\right\rangle\left(\left(\frac{\partial v_{l}}{\partial y^{j}}\right)^{\top, i}\left(\frac{\partial v_{l}}{\partial y^{k}}\right)^{\top, m}-\left(\frac{\partial v_{l}}{\partial y^{k}}\right)^{\top, i}\left(\frac{\partial v_{l}}{\partial y^{j}}\right)^{\top, m}\right), \\
\not \psi^{m}= & \frac{\partial v_{l}^{i}}{\partial y^{j}} v_{l}^{m} \phi_{\alpha}^{i} \gamma_{\alpha} \cdot \psi^{j}
\end{aligned}
$$


Denote

$$
\Omega_{i}^{m}:=\left(\begin{array}{c}
\lambda_{i}^{m} \\
\mu_{i}^{m}
\end{array}\right), \quad i, m=1, \ldots, K
$$

where

$$
\begin{aligned}
\lambda_{i}^{m}:= & \left(\frac{\partial v_{l}^{i}}{\partial y^{j}} v_{l}^{m}-\frac{\partial v_{l}^{m}}{\partial y^{j}} v_{l}^{i}\right) \phi_{1}^{j} \\
& +\left(\left(\frac{\partial v_{l}}{\partial y^{j}}\right)^{\top, i}\left(\frac{\partial v_{l}}{\partial y^{k}}\right)^{\top, m}-\left(\frac{\partial v_{l}}{\partial y^{k}}\right)^{\top, i}\left(\frac{\partial v_{l}}{\partial y^{j}}\right)^{\top, m}\right)\left\langle\psi^{k}, \gamma_{1} \cdot \psi^{j}\right\rangle, \\
\mu_{i}^{m}:= & \left(\frac{\partial v_{l}^{i}}{\partial y^{j}} v_{l}^{m}-\frac{\partial v_{l}^{m}}{\partial y^{j}} v_{l}^{i}\right) \phi_{2}^{j} \\
& +\left(\left(\frac{\partial v_{l}}{\partial y^{j}}\right)^{\top, i}\left(\frac{\partial v_{l}}{\partial y^{k}}\right)^{\top, m}-\left(\frac{\partial v_{l}}{\partial y^{k}}\right)^{\top, i}\left(\frac{\partial v_{l}}{\partial y^{j}}\right)^{\top, m}\right)\left\langle\psi^{k}, \gamma_{2} \cdot \psi^{j}\right\rangle .
\end{aligned}
$$

Then we can write (2.26) in the form

$$
-\Delta \phi^{m}=\Omega_{i}^{m} \cdot \nabla \phi^{i}
$$

It is easy to verify that $\Omega=\left(\Omega_{i}^{m}\right)_{1 \leq i, m \leq K} \in L^{2}\left(B_{1}, \operatorname{so}(K) \otimes \mathbb{R}^{2}\right)$. By Theorem A, we have $\phi \in C^{0}\left(B_{1}, N\right)$, and consequently $(\phi, \psi)$ is smooth.

\section{Free boundary problem for Dirac-harmonic maps}

In this section, we shall study the free boundary problem for Dirac-harmonic maps.

First, we impose the free boundary condition for the map in the classical sense, namely, the boundary of the domain is mapped freely into a submanifold of the target. Next, motivated by the supersymmetric sigma model with boundaries (see AlbertssonLindström-Zabzine [1, 2]), we impose the boundary condition for the spinor field using a chirality operator.

To begin, let us recall the chirality boundary conditions for the usual Dirac operator $\not \partial$ (see [16]).

\section{Chirality boundary conditions for the Dirac operator $\not \partial$}

Let $M$ be a compact Riemannian spin surface with boundary $\partial M \neq \emptyset$. Then $M$ admits a chirality operator $G=\gamma\left(\omega_{2}\right)$, the Clifford multiplication by the complex volume form $\omega_{2}=i \gamma_{1} \gamma_{2}$. The operator $G$ is an endomorphism of the spinor bundle $\Sigma M$ satisfying

$$
\begin{aligned}
& G^{2}=I,\langle G \psi, G \varphi\rangle=\langle\psi, \varphi\rangle, \\
& \nabla_{X}(G \psi)=G \nabla_{X} \psi, \quad X \cdot G \psi=-G(X \cdot \psi),
\end{aligned}
$$

for all $X \in \Gamma(T M)$ and $\psi, \varphi \in \Gamma(\Sigma M)$. Here $I$ denotes the identity endomorphism of $\Sigma M$. 
Denote by

$$
\mathbf{S}:=\left.\Sigma M\right|_{\partial M}
$$

the restricted spinor bundle with induced Hermitian product.

Let $\vec{n}$ be the outward unit normal vector field on $\partial M$. One can verify that $\vec{n} G$ : $\Gamma(\mathbf{S}) \rightarrow \Gamma(\mathbf{S})$ is a self-adjoint endomorphism whose square is the identity:

$$
\begin{aligned}
\langle\vec{n} G \psi, \varphi\rangle & =\langle\psi, \vec{n} G \varphi\rangle, \\
(\vec{n} G)^{2} & =I .
\end{aligned}
$$

Hence, we can decompose $\mathbf{S}=V^{+} \oplus V^{-}$, where $V^{ \pm}$is the eigensubbundle corresponding to the eigenvalue \pm 1 . One verifies that the orthogonal projection onto the eigensubbundle $V^{ \pm}$,

$$
\mathbf{B}^{ \pm}: L^{2}(\mathbf{S}) \rightarrow L^{2}\left(V^{ \pm}\right), \quad \psi \mapsto \frac{1}{2}(I \pm \vec{n} G) \psi,
$$

defines a local elliptic boundary condition for the Dirac operator $\not \partial$ (see [16]). We say a spinor $\psi \in W^{1,4 / 3}(\Sigma M)$ satisfies the boundary condition $\mathbf{B}^{ \pm}$if

$$
\left.\mathbf{B}^{ \pm} \psi\right|_{\partial M}=0
$$

The following proposition was shown in [16]. For completeness, we present the proof using our notations.

Proposition 3.1. If $\varphi, \psi \in W^{1,4 / 3}(\Sigma M)$ satisfy the boundary condition $\mathbf{B}^{ \pm}$then

$$
\langle\vec{n} \cdot \psi, \varphi\rangle=0 \quad \text { on } \partial M \text {. }
$$

In particular,

$$
\int_{\partial M}\langle\vec{n} \cdot \psi, \varphi\rangle=0 .
$$

Proof. Let $\varphi, \psi \in W^{1,4 / 3}(\Sigma M)$ satisfy the boundary condition $\mathbf{B}^{ \pm}$, i.e., $\left.\mathbf{B}^{ \pm} \psi\right|_{\partial M}=$ $\left.\mathbf{B}^{ \pm} \varphi\right|_{\partial M}=0$. Then

$$
\vec{n} G \psi=\mp \psi, \quad \vec{n} G \varphi=\mp \varphi
$$

Hence, applying the properties (3.28)-(3.31) of $G$, we get

$\langle\vec{n} \cdot \psi, \varphi\rangle=\langle G \vec{n} \cdot \psi, G \varphi\rangle=\langle-\vec{n} G \psi,-\vec{n} \vec{n} G \varphi\rangle=(-1)^{2}(\mp 1)^{2}\langle\psi, \vec{n} \varphi\rangle=-\langle\vec{n} \cdot \psi, \varphi\rangle$.

Now (3.33) and (3.34) follow immediately.

Let $M$ be the upper-half Euclidean space $\mathbb{R}_{+}^{2}$. We identify the Clifford multiplication by the orthonormal frame $\partial x_{1}, \partial x_{2}$ with the following matrices:

$$
\gamma_{1}=\left(\begin{array}{ll}
0 & i \\
i & 0
\end{array}\right), \quad \gamma_{2}=\left(\begin{array}{cc}
0 & 1 \\
-1 & 0
\end{array}\right)
$$


Then we can take the chirality operator $G:=i \gamma_{1} \gamma_{2}=\left(\begin{array}{cc}1 & 0 \\ 0 & -1\end{array}\right)$. Note that $\vec{n}=-\partial x_{2}=$ $-\gamma_{2}=\left(\begin{array}{cc}0 & -1 \\ 1 & 0\end{array}\right)$, and hence we can calculate $\mathbf{B}^{ \pm}=\frac{1}{2}(I \pm \vec{n} \cdot G)=\frac{1}{2}\left(\begin{array}{cc}1 & \pm 1 \\ \pm 1 & 1\end{array}\right)$.

By the standard chirality decomposition, we can write $\psi=\left(\begin{array}{l}\psi_{+} \\ \psi_{-}\end{array}\right)$; then the boundary condition (3.32) becomes

$$
\psi_{+}=\mp \psi_{-} \quad \text { on } \partial M .
$$

Next, we will extend the chirality boundary condition to the Dirac operator along a map.

Chirality boundary condition for the Dirac operator ID along a map $\phi$

When $\partial M \neq \emptyset$, the Dirac operator $\not D$ along a map $\phi$ is in general not formally self-adjoint. In fact, we have the following property analogous to one for the usual Dirac operator $\not \partial$.

\section{Proposition 3.2.}

$$
\int_{M}\langle\psi, \not D \varphi\rangle=\int_{M}\langle\not D \psi, \varphi\rangle-\int_{\partial M}\langle\vec{n} \cdot \psi, \varphi\rangle
$$

for all $\psi, \varphi \in C^{\infty}\left(\Sigma M \otimes \phi^{-1} T N\right)$, where $\langle\psi, \varphi\rangle:=g_{i j}(\phi)\left\langle\psi^{i}, \varphi^{j}\right\rangle$.

Proof. Choose a local orthonormal frame $\left\{\gamma_{\alpha}\right\}_{\alpha=1}^{2}$ on $M$. Given $\psi, \varphi \in C^{\infty}(\Sigma M \otimes$ $\left.\phi^{-1} T N\right)$, define

$$
f:=\left\langle\gamma_{\alpha} \cdot \psi, \varphi\right\rangle \gamma_{\alpha} ;
$$

then $f$ is independent of the choice of such a frame $\gamma_{\alpha}$ and hence is globally defined. We calculate

$$
\begin{aligned}
\int_{M}\langle\psi, \not D \varphi\rangle & =\int_{M}\langle\not D \psi, \varphi\rangle-\int_{M} \gamma_{\alpha}\left\langle\gamma_{\alpha} \cdot \psi, \varphi\right\rangle=\int_{M}\langle\not D \psi, \varphi\rangle-\int_{M} \operatorname{div} f \\
& =\int_{M}\langle\not D \psi, \varphi\rangle-\int_{\partial M} f \cdot \vec{n}=\int_{M}\langle\not D \psi, \varphi\rangle-\int_{\partial M}\left\langle\gamma_{\alpha} \cdot \psi, \varphi\right\rangle\left\langle\gamma_{\alpha}, \vec{n}\right\rangle \\
& =\int_{M}\langle\not D \psi, \varphi\rangle-\int_{\partial M}\langle\vec{n} \cdot \psi, \varphi\rangle .
\end{aligned}
$$

Here in the last step we have used the fact that $\vec{n}=\left\langle\gamma_{\alpha}, \vec{n}\right\rangle \gamma_{\alpha}$.

To extend the chirality boundary condition to the Dirac operator $\not D$ along a map from $M$ to $N$, we need some geometric structure on the $\operatorname{target} N$.

Given a submanifold $\mathcal{S}$ of $N$, we assume that there is an endomorphism $R(y)$ : $T_{y} N \rightarrow T_{y} N$ for all $y \in \mathcal{S}$. The $(1,1)$ tensor $R$ is called compatible if it preserves the metric on $T N$, that is,

$$
\langle R(y) V, R(y) W\rangle=\langle V, W\rangle, \quad \forall V, W \in T_{y} N, \forall y \in \mathcal{S}
$$


and it squares to the identity, more precisely,

$$
R(y) R(y) V=V, \quad \forall V \in T_{y} N, \forall y \in \mathcal{S} .
$$

Such compatible $(1,1)$ tensors on $\mathcal{S}$ always exist. For instance, we can take $R \equiv \pm \mathrm{id}$, where

$$
\text { id }: T_{y} N \rightarrow T_{y} N, \quad \forall y \in \mathcal{S},
$$

denotes the identity endomorphism.

Let $\mathcal{S}$ be a closed submanifold of $N$ with a compatible $(1,1)$ tensor $R$ and consider a $\operatorname{map} \phi \in C^{\infty}(M, N)$ satisfying the free boundary condition in the classical sense, that is, $\phi(\partial M) \subset \mathcal{S}$. We denote by

$$
\mathbf{S}_{\phi}:=\left.\left(\Sigma M \otimes \phi^{-1} T N\right)\right|_{\partial M}
$$

the restricted (twisted) spinor bundle with the induced metric.

Let $\psi \in C^{\infty}\left(\mathbf{S}_{\phi}\right)$. Given $x \in \partial M$, we have $\phi(x) \in \mathcal{S}$. Choose a local orthonormal frame $\left\{V_{i}\right\}$ on a neighborhood of $\phi(x)$ (still denote by $\left\{V_{i}\right\}$ the corresponding orthonormal frame along the map $\phi)$. Locally, we can write

$$
\psi=\sum_{i} \psi^{i} \otimes V_{i}
$$

Denote by Id the identity endomorphism acting on $C^{\infty}\left(\left.\phi^{-1} T N\right|_{\partial M}\right)$. Then, one can verify that the endomorphism $\vec{n} G \otimes R: C^{\infty}\left(\mathbf{S}_{\phi}\right) \rightarrow C^{\infty}\left(\mathbf{S}_{\phi}\right)$ defined by

$$
(\vec{n} G \otimes R) \psi:=\sum_{i} \vec{n} G \psi^{i} \otimes R V_{i}, \quad \forall \psi=\sum_{i} \psi^{i} \otimes V_{i} \in C^{\infty}\left(\mathbf{S}_{\phi}\right),
$$

is self-adjoint and its square is the identity:

$$
\begin{aligned}
\langle(\vec{n} G \otimes R) \psi, \varphi\rangle & =\langle\psi,(\vec{n} G \otimes R) \varphi\rangle, \quad \forall \psi, \varphi \in C^{\infty}\left(\mathbf{S}_{\phi}\right), \\
(\vec{n} G \otimes R)^{2} & =I \otimes \mathrm{Id} .
\end{aligned}
$$

Hence, we can decompose the twisted bundle $\mathbf{S}_{\phi}$ as $V_{\phi}^{+} \oplus V_{\phi}^{-}$, where $V_{\phi}^{ \pm}$is the eigensubbundle corresponding to the eigenvalue \pm 1 . One verifies that the orthogonal projection onto the eigensubbundle $V_{\phi}^{ \pm}$,

$$
\mathbf{B}_{\phi}^{ \pm}: C^{\infty}\left(\mathbf{S}_{\phi}\right) \rightarrow C^{\infty}\left(V_{\phi}^{ \pm}\right), \quad \psi \mapsto \frac{1}{2}(I \otimes \operatorname{Id} \pm \vec{n} G \otimes R) \psi
$$

defines an elliptic boundary condition for the Dirac operator $\not D$ along the map $\phi$. We say a spinor field $\psi \in C^{\infty}\left(\Sigma M \otimes \phi^{-1} T N\right)$ along a map $\phi$ satisfies the boundary condition $\mathbf{B}_{\phi}^{ \pm}$ if

$$
\left.\mathbf{B}_{\phi}^{ \pm} \psi\right|_{\partial M}=0
$$

The following proposition generalizes the results of Proposition 3.1 to the case of spinor fields along a map: 
Proposition 3.3. If $\varphi, \psi \in C^{\infty}\left(\Sigma M \otimes \phi^{-1} T N\right)$ satisfy the chirality boundary condition $\mathbf{B}_{\phi}^{ \pm}$, then

$$
\langle\vec{n} \cdot \psi, \varphi\rangle=0 \quad \text { on } \partial M .
$$

In particular,

$$
\int_{\partial M}\langle\vec{n} \cdot \psi, \varphi\rangle=0
$$

Proof. Let $\psi, \varphi \in C^{\infty}\left(\Sigma M \otimes \phi^{-1} T N\right)$ satisfy the chirality boundary condition $\mathbf{B}_{\phi}^{ \pm}$, that is, $\left.\mathbf{B}_{\phi}^{ \pm} \psi\right|_{\partial M}=\left.\mathbf{B}_{\phi}^{ \pm} \varphi\right|_{\partial M}=0$. Choosing a local orthonormal frame $\left\{V_{i}\right\}$ on a neighborhood of $\phi(x)$ for $x \in \partial M$, we can write

$$
\psi=\sum_{i} \psi^{i} \otimes V_{i}, \quad \varphi=\sum_{j} \varphi^{j} \otimes V_{j} .
$$

Then the chirality boundary conditions $\mathbf{B}_{\phi}^{ \pm}$for $\psi$ and $\varphi$ read

$$
\psi=\sum_{i} \psi^{i} \otimes V_{i}=\mp \sum_{i} \vec{n} G \psi^{i} \otimes R V_{i}, \quad \varphi=\sum_{j} \varphi^{j} \otimes V_{j}=\mp \sum_{j} \vec{n} G \varphi^{j} \otimes R V_{j} .
$$

At the point $x$, we can calculate

$$
\begin{aligned}
\langle\vec{n} \cdot \psi, \varphi\rangle & =(\mp 1)^{2} \sum_{i, j}\left\langle\vec{n} \vec{n} G \psi^{i} \otimes R V_{i}, \vec{n} G \varphi^{j} \otimes R V_{j}\right\rangle=\sum_{i, j}\left\langle\vec{n} \vec{n} G \psi^{i}, \vec{n} G \varphi^{j}\right\rangle\left\langle R V_{i}, R V_{j}\right\rangle \\
& =\sum_{i, j}\left\langle-\vec{n} \psi^{i}, \varphi^{j}\right\rangle\left\langle V_{i}, V_{j}\right\rangle=\sum_{i, j}-\left\langle\vec{n} \psi^{i} \otimes V_{i}, \varphi^{j} \otimes V_{j}\right\rangle=\sum_{i, j}-\langle\vec{n} \cdot \psi, \varphi\rangle .
\end{aligned}
$$

Since the point $x \in \partial M$ is arbitrary, we obtain (3.39) and (3.40).

\section{Free boundary conditions for Dirac-harmonic maps}

Let $\mathcal{S}$ be a closed $p$-dimensional submanifold of $N$. It turns out that one can associate to it a natural $(1,1)$ tensor $R$ that is compatible.

To see this, we consider a tubular neighborhood $\mathbf{U}_{\delta}:=\left\{z \in N \mid \operatorname{dist}^{N}(z, \mathcal{S})<\delta\right\}$ of $\mathcal{S}$ in $N$, where $\delta>0$ is a constant small enough such that for any $z \in \mathbf{U}_{\delta}$, there exists a unique minimal geodesic $\gamma_{z}$ connecting $z$ and $z^{\prime} \in \mathcal{S}$ which attains the distance from $z$ to the submanifold $\mathcal{S}$.

On $\mathbf{U}_{\delta}$, we can define the geodesic reflection $\sigma$ as follows:

$$
\sigma: \mathbf{U}_{\delta} \rightarrow \mathbf{U}_{\delta}, \quad z:=\exp _{z^{\prime}} v \mapsto \sigma(z):=\exp _{z^{\prime}}(-v),
$$

where $v \in T_{z^{\prime}} N$ is uniquely determined by $z$. Clearly, $\sigma^{2}=\mathrm{id}: \mathbf{U}_{\delta} \rightarrow \mathbf{U}_{\delta}$, and for $\delta$ small enough, the map $\sigma$ is a diffeomorphism. Associated to this $\sigma$, there is a $(1,1)$ tensor $R$ on $\mathcal{S}$ defined by

$$
R(z):=D \sigma(z), \quad \forall z \in \mathcal{S}
$$


The $(1,1)$ tensor $R$ is well defined on $\mathcal{S}$, since $\left.\sigma\right|_{\mathcal{S}}=$ id and hence $R(z): T_{z} N \rightarrow$ $T_{z} N$ is an endomorphism for $z \in \mathcal{S}$. To show the compatibility of $R$, it is most convenient to take the adapted coordinates $\left\{y^{i}\right\}_{i=1, \ldots, d}$ in some neighborhood $\mathrm{U} \subset \mathbf{U}_{\delta}$ of a given point $P \in \mathcal{S}$, such that $\left\{y^{a}\right\}_{a=1, \ldots, p}$ are coordinates in $\mathcal{S},\left\{y^{\lambda}\right\}_{\lambda=p+1, \ldots, d}$ are the directions normal to $\mathcal{S}$ and

$$
\mathcal{S} \cap \mathrm{U}=\left\{y \in \mathrm{U} \mid y^{p+1}=\cdots=y^{d}=0\right\} .
$$

In what follows, the index ranges are:

$$
1 \leq a, b, \ldots \leq p, \quad p+1 \leq \lambda, \mu, \ldots \leq d, \quad 1 \leq i, j, k, \ldots \leq d
$$

Note that the adapted coordinates $\left\{y^{i}\right\}_{i=1, \ldots, d}$ are exactly the geodesic parallel coordinates for the submanifold $\mathcal{S}$. These coordinates also go under the name of Fermi coordinates in the literature. We refer to [12] for more details. In such coordinates, the diffeomorphism $\left.\sigma\right|_{\mathrm{U}}: \mathrm{U} \rightarrow \mathrm{U}$ is given by

$$
\sigma:\left(y^{1}, \ldots, y^{p}, y^{p+1}, \ldots, y^{d}\right) \rightarrow\left(y^{1}, \ldots, y^{p},-y^{p+1}, \ldots,-y^{d}\right) .
$$

Consequently, we have

$$
\begin{array}{rlrl}
D \sigma\left(\partial y^{k}\right) & =\partial y^{k}, & k & =1, \ldots, p, \\
D \sigma\left(\partial y^{m}\right) & =-\partial y^{m}, \quad m & =p+1, \ldots, d .
\end{array}
$$

The tensor $R$ and the metric $g$ take the forms

$$
R=\left(\begin{array}{cc}
\delta_{b}^{a} & 0 \\
0 & -\delta_{\mu}^{\lambda}
\end{array}\right), \quad g=\left(\begin{array}{cc}
g_{a b} & 0 \\
0 & g_{\lambda \mu}
\end{array}\right)
$$

It is easy to verify that $R$ is compatible. Moreover, $R$ has the following additional property:

$$
\left.R(z)\right|_{T_{z} \mathcal{S}}=\mathrm{id},\left.\quad R(z)\right|_{T_{z}^{\perp \mathcal{S}}}=-\mathrm{id}, \quad \forall z \in \mathcal{S},
$$

where id denotes the identity endomorphism and $T_{z}^{\perp} \mathcal{S}$ is the subspace of $T_{z} N$ that is normal to $T_{z} \mathcal{S}$.

Given a closed $p$-dimensional submanifold $\mathcal{S}$ of $N$, we will always associate to it the compatible $(1,1)$ tensor $R$ constructed via the geodesic reflection $\sigma$ for $\mathcal{S}$. It turns out that this tensor is the most natural one from a geometrical and analytical point of view.

Let $\phi \in C^{\infty}(M, N)$ satisfy the boundary condition $\phi(\partial M) \subset \mathcal{S}$ and let $\psi \in$ $C^{\infty}\left(\Sigma M \otimes \phi^{-1} T N\right)$. We impose the free boundary condition for $\psi$ to be the chirality boundary condition corresponding to $\mathcal{S}$,

$$
\left.\mathbf{B}_{\phi}^{ \pm} \psi\right|_{\partial M}=0
$$

or in local form

$$
\psi^{i}=\mp R_{j}^{i} \vec{n} G \psi^{j}, \quad i=1, \ldots, d, \quad \text { on } \partial M \text {. }
$$


When $M=\mathbb{R}_{+}^{2}$, we identify the Clifford multiplication by $\partial x_{1}, \partial x_{2}$ with the matrices $\gamma_{1}, \gamma_{2}$, take the chirality operator $G:=i \gamma_{1} \gamma_{2}$ and decompose $\psi=\left(\begin{array}{l}\psi_{+} \\ \psi_{-}\end{array}\right)$. Then the chirality boundary condition $\mathbf{B}_{\phi}^{ \pm}$corresponding to $\mathcal{S}$ becomes

$$
\psi_{+}^{i}=\mp R_{j}^{i} \psi_{-}^{j}, \quad i=1, \ldots, d, \quad \text { on } \partial M .
$$

Remark 3.1. In the physics literature (see [1]), the above coordinate system $\left\{y^{i}\right\}_{i=1, \ldots, d}$ is said to be adapted to the brane $\mathcal{S}$. And (3.41) is the fermionic boundary condition considered in [1], where it is a priori assumed that there exists some compatible $(1,1)$ tensor $R$ defined on some region including $\mathcal{S}$.

Set

$\mathcal{X}(M, N ; \mathcal{S})$

$:=\left\{(\phi, \psi)\left|\phi \in C^{\infty}(M, N), \phi(\partial M) \subset \mathcal{S} ; \psi \in C^{\infty}\left(\Sigma M \otimes \phi^{-1} T N\right), \mathbf{B}_{\phi}^{ \pm} \psi\right|_{\partial M}=0\right\}$.

Definition 3.1. $(\phi, \psi) \in \mathcal{X}(M, N ; \mathcal{S})$ is called a Dirac-harmonic map from $M$ to $N$ with free boundary on $\mathcal{S}$ if it is a critical point of $L(\cdot, \cdot)$ in $\mathcal{X}(M, N ; \mathcal{S})$.

Let $(\phi, \psi)$ be a Dirac-harmonic map from $M$ to $N$ with free boundary on $\mathcal{S} \subset N$.

First, we consider a family of $\left(\phi_{t}, \psi_{t}\right) \in \mathcal{X}(M, N ; \mathcal{S})$ with $\phi_{t} \equiv \phi$ and $\left.\frac{d \psi_{t}}{d t}\right|_{t=0}=\xi$. Then we calculate

$$
\begin{aligned}
\left.\frac{d L\left(\phi_{t}, \psi_{t}\right)}{d t}\right|_{t=0} & =\left.\int_{M} \frac{d}{d t}\left\langle\psi_{t}, \not D \psi_{t}\right\rangle\right|_{t=0}=\int_{M}\langle\xi, \not D \psi\rangle+\int_{M}\langle\psi, \not D \xi\rangle \\
& =2 \int_{M} \operatorname{Re}\langle\xi, \not D \psi\rangle-\int_{\partial M}\langle\vec{n} \cdot \psi, \xi\rangle .
\end{aligned}
$$

Note that $\psi, \xi$ satisfy the boundary condition $\mathbf{B}_{\phi}^{ \pm}$, hence Proposition 3.3 shows that $\int_{\partial M}\langle\vec{n} \cdot \psi, \xi\rangle=0$.

Next, we consider a family of $\left(\phi_{t}, \psi_{t}\right) \in \mathcal{X}(M, N ; \mathcal{S})$ with $\left.\frac{d \phi_{t}}{d t}\right|_{t=0}=\eta$ and $\psi_{t}=$ $\psi_{t}^{i} \otimes \partial y^{i}\left(\phi_{t}\right), \psi_{t}^{i} \equiv \psi^{i}$. Then

$$
\begin{aligned}
& \left.\frac{d L\left(\phi_{t}, \psi_{t}\right)}{d t}\right|_{t=0}=\int_{M} 2\langle d \phi, d \eta\rangle+\left.\int_{M}\left\langle\psi, \frac{d}{d t} \not D \psi_{t}\right\rangle\right|_{t=0} \\
& =\int_{M} 2\langle d \phi, d \eta\rangle+\int_{M} 2\langle R(\phi, \psi), \eta\rangle+\int_{M}\left\langle\psi, \not D\left(\psi^{i} \otimes \nabla_{\partial t} \partial y^{i}\right)\right\rangle \\
& =\int_{M} 2\langle-\tau(\phi), \eta\rangle+\int_{M} 2\langle R(\phi, \psi), \eta\rangle+\int_{M}\left\langle\not D \psi, \psi^{i} \otimes \nabla_{\partial t} \partial y^{i}\right\rangle \\
& +\int_{\partial M} 2\left\langle\phi_{\vec{n}}, \eta\right\rangle-\int_{\partial M}\left\langle\vec{n} \cdot \psi, \psi^{i} \otimes \nabla_{\partial t} \partial y^{i}\right\rangle .
\end{aligned}
$$


Here $\phi_{\vec{n}}=\partial \phi / \partial \vec{n}$. Note that, for simplicity, we used the local expression of $\psi$, namely, $\psi=\psi^{i} \otimes \partial y^{i}$, where $y^{i}$ is a local coordinate of $N$. By using the expression $\psi^{i} \otimes \nabla_{\partial t} \partial y^{i}=$ $\eta^{j} \Gamma_{j i}^{k} \psi^{i} \otimes \partial y^{k}$ and requiring the vanishing of the boundary integral, we have

$0=\int_{\partial M} 2\left\langle\phi_{\vec{n}}, \eta\right\rangle-\int_{\partial M}\left\langle\vec{n} \cdot \psi, \psi^{i} \otimes \nabla_{\partial t} \partial y^{i}\right\rangle=\int_{\partial M} g_{m j}\left(2 \phi_{\vec{n}}^{m}-g^{n m}\left\langle\vec{n} \cdot \psi^{l}, \psi^{i}\right\rangle \Gamma_{i n}^{k} g_{k l}\right) \eta^{j}$.

Since $\eta=\left.\frac{d \phi_{t}}{d t}\right|_{t=0}$ is arbitrary, it follows that

$$
\left(2 \phi_{\vec{n}}^{m}-g^{n m}\left\langle\vec{n} \cdot \psi^{l}, \psi^{i}\right\rangle \Gamma_{i n}^{k} g_{k l}\right) \partial_{m} \perp \mathcal{S}
$$

here and below, for simplicity, we write $\partial_{i}:=\partial / \partial y^{i}, \partial_{\lambda}:=\partial / \partial y^{\lambda}, \partial_{a}:=\partial / \partial y^{a}$ etc.

From the free boundary conditions for the spinor fields,

$$
\psi^{i}=\mp R_{j}^{i} \vec{n} G \psi^{j} \quad \text { on } \partial M
$$

where $R=\left(R_{j}^{i}\right)=\left(\begin{array}{cc}\delta_{b}^{a} & 0 \\ 0 & -\delta_{\mu}^{\lambda}\end{array}\right)$, one easily verifies that

$$
\left\langle\vec{n} \cdot \psi^{a}, \psi^{b}\right\rangle=0, \quad\left\langle\vec{n} \cdot \psi^{\lambda}, \psi^{\mu}\right\rangle=0, \quad \text { on } \partial M,
$$

for $a, b=1, \ldots, p$ and $\lambda, \mu=p+1, \ldots, d$.

Let us continue to consider (3.42). We note that

$$
\begin{aligned}
g^{m n} g_{k l} \Gamma_{i n}^{k}\left\langle\vec{n} \cdot \psi^{l}, \psi^{i}\right\rangle & =g^{m n} g_{\lambda l} \Gamma_{i n}^{\lambda}\left\langle\vec{n} \cdot \psi^{l}, \psi^{i}\right\rangle+g^{m n} g_{a l} \Gamma_{i n}^{a}\left\langle\vec{n} \cdot \psi^{l}, \psi^{i}\right\rangle \\
& =g^{m n} g_{\lambda \mu} \Gamma_{i n}^{\lambda}\left\langle\vec{n} \cdot \psi^{\mu}, \psi^{i}\right\rangle+g^{m n} g_{a b} \Gamma_{i n}^{a}\left\langle\vec{n} \cdot \psi^{b}, \psi^{i}\right\rangle \\
& =g^{m n} g_{\lambda \mu} \Gamma_{a n}^{\lambda}\left\langle\vec{n} \cdot \psi^{\mu}, \psi^{a}\right\rangle+g^{m n} g_{a b} \Gamma_{\lambda n}^{a}\left\langle\vec{n} \cdot \psi^{b}, \psi^{\lambda}\right\rangle \\
& =g^{m n} g_{\lambda \mu} \Gamma_{a n}^{\lambda}\left\langle\vec{n} \cdot \psi^{\mu}, \psi^{a}\right\rangle+g^{m n} g_{\lambda \mu} \Gamma_{b n}^{\mu}\left\langle\psi^{b}, \vec{n} \cdot \psi^{\lambda}\right\rangle,
\end{aligned}
$$

so that

$$
g^{m n} g_{k l} \Gamma_{i n}^{k}\left\langle\vec{n} \cdot \psi^{l}, \psi^{i}\right\rangle=2 g^{m n} g_{\lambda \mu} \Gamma_{a n}^{\lambda}\left\langle\vec{n} \cdot \psi^{\mu}, \psi^{a}\right\rangle, \quad m=1, \ldots, d .
$$

Using this we have

$$
\begin{aligned}
\left(2 \phi_{\vec{n}}^{m}-g^{n m}\left\langle\vec{n} \cdot \psi^{l}, \psi^{i}\right\rangle \Gamma_{i n}^{k} g_{k l}\right) \partial_{m} \perp \mathcal{S} & \Leftrightarrow\left(2 \phi_{\vec{n}}^{c}-g^{n c}\left\langle\vec{n} \cdot \psi^{l}, \psi^{i}\right\rangle \Gamma_{i n}^{k} g_{k l}\right) \partial_{c}=0 \\
& \Leftrightarrow 2 \phi_{\vec{n}}^{c} \partial_{c}-g^{d c}\left\langle\vec{n} \cdot \psi^{l}, \psi^{i}\right\rangle \Gamma_{i d}^{k} g_{k l} \partial_{c}=0 \\
& \Leftrightarrow\left(\frac{\partial \phi}{\partial \vec{n}}\right)^{\top}-g^{c d} \Gamma_{a d}^{\lambda} g_{\lambda \mu}\left\langle\vec{n} \cdot \psi^{\mu}, \psi^{a}\right\rangle \partial_{c}=0 .
\end{aligned}
$$

On the other hand, for the second fundamental form $A_{\mathcal{S}}(\cdot, \cdot)$ of $\mathcal{S}$ in $N$, we have $A_{\mathcal{S}}\left(\partial_{a}, \partial_{d}\right)=\left(\nabla_{\partial_{a}} \partial_{d}\right)^{\perp}=\Gamma_{a d}^{\mu} \partial_{\mu}$; using this in (3.42), we obtain

$$
\begin{aligned}
\left(2 \phi_{\vec{n}}^{m}-g^{n m}\left\langle\vec{n} \cdot \psi^{l}, \psi^{i}\right\rangle \Gamma_{i n}^{k} g_{k l}\right) \partial_{m} \perp \mathcal{S} & \\
\Leftrightarrow\left(\frac{\partial \phi}{\partial \vec{n}}\right)^{\top} & =g^{c d}\left\langle A_{\mathcal{S}}\left(\partial_{a}, \partial_{d}\right), \partial_{\mu}\right\rangle\left\langle\vec{n} \cdot \psi^{\mu}, \psi^{a}\right\rangle \partial_{c}=g^{c d}\left\langle A_{\mathcal{S}}\left(\psi^{\top}, \partial_{d}\right), \vec{n} \cdot \psi^{\perp}\right\rangle \partial_{c} \\
& =g^{c d}\left\langle P_{\mathcal{S}}\left(\vec{n} \cdot \psi^{\perp} ; \psi^{\top}\right), \partial_{d}\right\rangle \partial_{c}=P_{\mathcal{S}}\left(\vec{n} \cdot \psi^{\perp} ; \psi^{\top}\right) .
\end{aligned}
$$

Here $P_{\mathcal{S}}(\cdot ; \cdot)$ is the shape operator of $\mathcal{S}$ in $N$. Therefore, we have 
Proposition 3.4. The condition (3.42) is equivalent to

$$
\left(\frac{\partial \phi}{\partial \vec{n}}\right)^{\top}=P_{\mathcal{S}}\left(\vec{n} \cdot \psi^{\perp} ; \psi^{\top}\right) ;
$$

in particular, if $\mathcal{S}$ is a totally geodesic submanifold in $N$, this reads

$$
\frac{\partial \phi}{\partial \vec{n}} \perp \mathcal{S}
$$

Remark 3.2. The condition $\frac{\partial \phi}{\partial \vec{n}} \perp \mathcal{S}$ is exactly the orthogonality condition in the theory of minimal surfaces with free boundaries (see the survey paper by Hildebrandt [17] and the references therein). In the case of Dirac-harmonic maps with free boundaries, the orthogonality condition appears when the supporting submanifold $\mathcal{S}$ is totally geodesic or the spinor field vanishes, i.e. $\psi \equiv 0$.

The above discussion leads to the following equivalent definition of Dirac-harmonic maps with a free boundary on $\mathcal{S}$.

Definition 3.2. $(\phi, \psi) \in \mathcal{X}(M, N ; \mathcal{S})$ is called a Dirac-harmonic map from $M$ to $N$ with free boundary $\mathcal{S} \subset N$ if $(\phi, \psi)$ is Dirac-harmonic in $M$, i.e.,

$$
\tau(\phi)=R(\phi, \psi), \quad \not D \psi=0,
$$

and satisfies the following free boundary conditions:

(i) $\left(\frac{\partial \phi}{\partial \vec{n}}\right)^{\top}=P_{\mathcal{S}}\left(\vec{n} \cdot \psi^{\perp} ; \psi^{\top}\right)$ on $\partial M$,

(ii) $\left.\mathbf{B}_{\phi}^{ \pm} \psi\right|_{\partial M}=0$.

\section{Weakly Dirac-harmonic maps with free boundary on $\mathcal{S}$}

In order to define the free boundary conditions for weakly Dirac-harmonic maps, we shall use the isometric embedding $N \hookrightarrow \mathbb{R}^{K}$. Making use of the orthogonal decomposition $\mathbb{R}_{y}^{K}=T_{y} N \oplus T_{y}^{\perp} N$ for any $y \in N$, we can consider the bundles $\Sigma M \otimes \phi^{-1} T N$ and $\mathbf{S}_{\phi}=\left.\left(\Sigma M \otimes \phi^{-1} T N\right)\right|_{\partial M}$ as subbundles of $\Sigma M \otimes \phi^{-1} \mathbb{R}^{K}$ and $\left.\left(\Sigma M \otimes \phi^{-1} \mathbb{R}^{K}\right)\right|_{\partial M}$, respectively. Moreover, we denote

$$
L^{2}\left(\mathbf{S}_{\phi}\right):=\left\{\left.\psi\right|_{\partial M} \mid \psi \in W^{1,4 / 3}\left(\Sigma M \otimes \phi^{-1} T N\right)\right\} .
$$

Let $V_{\delta} N$ be a tubular neighborhood of $N$ in $\mathbb{R}^{K}$ with a projection $P: V_{\delta} N \rightarrow N$ (see [15]). We define

$$
\widetilde{R}(y):=D(\sigma \circ P)(y), \quad y \in \mathcal{S} .
$$

For $y \in \mathcal{S}$, since $R(y)=D \sigma(y)$, we have $\widetilde{R}(y)=D(\sigma \circ P)(y)=D \sigma(y) \circ(D P)(y)=$ $R(y) \circ(D P)(y)$. Moreover, for all $V, W \in T_{y} N$ and $y \in \mathcal{S}$, we have $D P(y) V=V$ and hence

$$
\begin{aligned}
\langle\widetilde{R}(y) V, \widetilde{R}(y) W\rangle_{\mathbb{R}_{y}^{K}} & =\langle R(y)[(D P)(y) V], R(y)[(D P)(y) W]\rangle_{\mathbb{R}_{y}^{K}} \\
& =\langle R(y) V, R(y) W\rangle_{T_{y} N}=\langle V, W\rangle_{T_{y} N}=\langle V, W\rangle_{\mathbb{R}_{y}^{K}} .
\end{aligned}
$$


On the other hand, since $(\sigma \circ P) \circ(\sigma \circ P)=\sigma \circ \sigma \circ P=P=$ id on $\mathbf{U}_{\delta} \subset N$, we get

$$
\widetilde{R}(y) \widetilde{R}(y) V=V, \quad \forall V \in T_{y} N, \forall y \in \mathcal{S} .
$$

Therefore, we can define, in analogy to the case of smooth sections, an endomorphism

$$
\vec{n} G \otimes \widetilde{R}: L^{2}\left(\mathbf{S}_{\phi}\right) \rightarrow L^{2}\left(\mathbf{S}_{\phi}\right),
$$

which is self-adjoint and squares to the identity. Also, we can decompose $\mathbf{S}_{\phi}=V_{\phi}^{+} \oplus V_{\phi}^{-}$ and define an elliptic boundary condition

$$
\widetilde{\mathbf{B}}_{\phi}^{ \pm}: L^{2}\left(\mathbf{S}_{\phi}\right) \rightarrow L^{2}\left(V_{\phi}^{ \pm}\right)
$$

for $\not D$. For convenience, we still denote $\widetilde{\mathbf{B}}_{\phi}^{ \pm}$by $\mathbf{B}_{\phi}^{ \pm}$.

One easily verifies that the results in Proposition 3.3 hold for $W^{1,4 / 3}$ sections of the bundle $\Sigma M \otimes \phi^{-1} T N$ with $\phi \in H^{1}(M, N)$. More precisely, we have

Proposition 3.5. If $\varphi, \psi \in W^{1,4 / 3}\left(\Sigma M \otimes \phi^{-1} T N\right)$ satisfy the chirality boundary condition $\mathbf{B}_{\phi}^{ \pm}$, then

$$
\langle\vec{n} \cdot \psi, \varphi\rangle=0 \quad \text { a.e. on } \partial M .
$$

In particular, $\int_{\partial M}\langle\vec{n} \cdot \psi, \varphi\rangle=0$.

Now we introduce the class $\mathcal{X}_{1,4 / 3}^{1,2}(M, N ; \mathcal{S})$ of admissible fields $(\phi, \psi)$ with free boundary on the supporting submanifold $\mathcal{S} \subset N$ as follows:

$$
\begin{aligned}
\mathcal{X}_{1,4 / 3}^{1,2}(M, N ; \mathcal{S}):=\left\{(\phi, \psi) \mid \phi \in H^{1}(M, N), \phi(\partial M) \subset \mathcal{S}\right. & \\
& \left.\psi \in W^{1,4 / 3}\left(\Sigma M \otimes \phi^{-1} T N\right),\left.\mathbf{B}_{\phi}^{ \pm} \psi\right|_{\partial M}=0\right\}
\end{aligned}
$$

where " $\phi(\partial M) \subset \mathcal{S}$ " means that the $L^{2}$-trace $\left.\phi\right|_{\partial M}$ of $\phi$ maps $\mathcal{H}^{1}$-almost all of $\partial M$ into $\mathcal{S}$, and " $\left.\mathbf{B}_{\phi}^{ \pm} \psi\right|_{\partial M}=0$ " means that the $L^{2}$-traces $\left.\mathbf{B}_{\phi}^{ \pm} \psi\right|_{\partial M}$ vanish on $\mathcal{H}^{1}$-almost all of $\partial M$.

Definition 3.3. An element $(\phi, \psi)$ of $\mathcal{X}_{1,4 / 3}^{1,2}(M, N ; \mathcal{S})$ is called a weakly Dirac-harmonic map with free boundary on $\mathcal{S}$ if it is a critical point of the action functional $L(\cdot, \cdot)$ in $\mathcal{X}_{1,4 / 3}^{1,2}(M, N ; \mathcal{S})$.

One verifies, similarly to Wang-Xu [28], that a Dirac-harmonic map with free boundary on $\mathcal{S}$ is invariant under a totally geodesic, isometric embedding of the target. Therefore, adapting Hélein's enlargement argument (see [14, 15]), we can assume that there exists a global orthonormal frame $\left\{\widehat{V}_{i}\right\}_{i=1}^{d}$ on $N$. Set $V_{i}(x)=\widehat{V}_{i}(\phi(x)), i=1, \ldots, d$; then $\left\{V_{i}\right\}$ is an orthonormal frame along the map $\phi$. The spinor field $\psi$ along $\phi$ can be written as

$$
\psi=\sum_{i=1}^{d} \psi^{i} \otimes V_{i} .
$$

Using the frame $\left\{\widehat{V}_{i}\right\}_{i=1}^{d}$, it is not difficult to derive (similarly to the calculations in $[6,28]$ ) the following two propositions (proofs omitted): 
Proposition 3.6. Let $(\phi, \psi) \in \mathcal{X}_{1,4 / 3}^{1,2}(M, N)$ be a weakly Dirac-harmonic map. Then

$$
\int_{M} d \phi \cdot \nabla V+\int_{M}\left\langle\psi^{i}, \gamma_{\alpha} \cdot \psi^{j}\right\rangle\left\langle V_{i}, R(\phi)\left(V, \phi_{*}\left(\gamma_{\alpha}\right)\right) V_{j}\right\rangle=0, \quad \int_{M}\langle\psi, \not D \xi\rangle=0,
$$

for all compactly supported $V \in H^{1} \cap L^{\infty}\left(M, \phi^{-1} T N\right)$ and for all compactly supported $\xi \in W^{1,4 / 3} \cap L^{\infty}\left(\Sigma M \otimes \phi^{-1} T N\right)$.

Proposition 3.7. Let $(\phi, \psi) \in \mathcal{X}_{1,4 / 3}^{1,2}(M, N ; \mathcal{S})$ be a weakly Dirac-harmonic map with free boundary on $\mathcal{S}$. Then

$$
\int_{M} d \phi \cdot \nabla V+\int_{M}\left\langle\psi^{i}, \gamma_{\alpha} \cdot \psi^{j}\right\rangle\left\langle V_{i}, R(\phi)\left(V, \phi_{*}\left(\gamma_{\alpha}\right)\right) V_{j}\right\rangle=0, \quad \int_{M}\langle\psi, \not D \xi\rangle=0,
$$

for all $V \in H^{1} \cap L^{\infty}\left(M, \phi^{-1} T N\right)$ such that $V(x) \in T_{\phi(x)} \mathcal{S}$ for a.e. $x \in \partial M$ and for all $\xi \in W^{1,4 / 3} \cap L^{\infty}\left(\Sigma M \otimes \phi^{-1} T N\right)$ such that $\left.\mathbf{B}_{\phi}^{ \pm} \xi\right|_{\partial M}=0$.

The rest of this section will be devoted to studying the regularity of weakly Dirac-harmonic maps with free boundary on $\mathcal{S}$. For simplicity, we will set our problem in a small neighborhood of a boundary point. To this end, we consider the case that the domain $M$ is $B_{1}^{+}:=\left\{\left(x_{1}, x_{2}\right) \in \mathbb{R}^{2} \mid x_{1}^{2}+x_{2}^{2} \leq 1, x_{2} \geq 0\right\}$ and the free boundary portion is $I:=\left\{\left(x_{1}, 0\right) \in \mathbb{R}^{2} \mid-1 \leq x_{1} \leq 1\right\}$. Moreover, we identify $\partial x_{\alpha}$ with $\gamma_{\alpha}, \alpha=1,2$.

\section{The reflection principle}

The following lemma, analogous to Lemma 3.1 in [26], shows that the image of $\phi$ over a sufficiently small neighborhood of a boundary point is contained in a tubular neighborhood of the supporting submanifold $\mathcal{S}$. Therefore, we can use the geodesic reflection $\sigma$ to reflect the two fields $(\phi, \psi)$ across $\mathcal{S}$ when restricted to a sufficiently small domain.

Lemma 3.1. Let $N$ be a compact Riemannian manifold, isometrically embedded in $\mathbb{R}^{K}$, and $\mathcal{S}$ a closed submanifold in $N$. Then there is an $\epsilon_{0}=\epsilon_{0}(N)>0$ such that for all weakly Dirac-harmonic maps $(\phi, \psi) \in \mathcal{X}_{1,4 / 3}^{1,2}\left(B_{1}^{+}, N ; \mathcal{S}\right)$ with free boundary on $\mathcal{S}$ and

$$
\int_{B_{1}^{+}}\left(|d \phi|^{2}+|\psi|^{4}\right) \leq \epsilon_{0}
$$

we have $\operatorname{dist}(\phi(x), \mathcal{S}) \leq C \epsilon_{0}^{1 / 2}$ for all $x \in B_{1 / 4}^{+}$with a constant $C=C(N)$. Moreover, there is a $Q \in \mathcal{S}$ such that $\phi(x) \in B_{C \epsilon_{0}^{1 / 2}}(Q)$ for all $x \in B_{1 / 4}^{+}$, with a constant $C=C(N)$.

Proof. To prove this lemma, it is sufficient to prove an interior estimate for Dirac-harmonic maps on surfaces. More precisely, let $x_{0} \in B_{1 / 4}^{+} \backslash \partial \mathbb{R}_{+}^{2}$ and set $R:=\frac{1}{3} \operatorname{dist}\left(x_{0}, \partial \mathbb{R}_{+}^{2}\right)$. Given $x \in B_{2 R}\left(x_{0}\right)$, one can verify that $B_{R}(x) \subset B_{1}^{+}$. Define

$$
\widetilde{\phi}(z):=\phi(x+R z), \quad \widetilde{\psi}(z):=R^{1 / 2} \psi(x+R z), \quad z \in B_{1} .
$$


Then by assumption (3.43), we have

$$
\int_{B_{1}}\left(|d \widetilde{\phi}|^{2}+|\widetilde{\psi}|^{4}\right)=\int_{B_{R}(x)}\left(|d \phi|^{2}+|\psi|^{4}\right) \leq \int_{B_{1}^{+}}\left(|d \phi|^{2}+|\psi|^{4}\right) \leq \epsilon_{0} .
$$

Provided that $\epsilon_{0}$ is sufficiently small, we can apply the $\epsilon$-regularity for Dirac-harmonic maps from surfaces (see [7, Theorem 3.2] or [6, Theorem 4.3]) to get

$$
\|d \widetilde{\phi}\|_{L^{\infty}\left(B_{1 / 2}\right)} \leq C\|d \widetilde{\phi}\|_{L^{2}\left(B_{1}\right)} \leq C \sqrt{\epsilon_{0}}
$$

where $C>0$ is a constant depending only on the geometry of $N$. Note that $d \widetilde{\phi}(0)=$ $R \cdot d \phi(x)$. Hence,

$$
|d \phi(x)|=\frac{|d \tilde{\phi}(0)|}{R} \leq \frac{C \sqrt{\epsilon_{0}}}{R} \quad \text { for all } x \in B_{2 R}\left(x_{0}\right) .
$$

Now we can use the same arguments as in the proof of [26, Lemma 3.1] to obtain

$$
\left|\phi\left(x_{0}\right)-\bar{\phi}\right| \leq C \sqrt{\epsilon_{0}}
$$

and

$$
\operatorname{dist}(\bar{\phi}, \mathcal{S}) \leq C\left[R^{2-n} \int_{B_{5 R}^{+}\left(x_{1}\right)}|d \phi|^{2}\right]^{1 / 2} \leq C \sqrt{\epsilon_{0}}, \quad \text { where } \quad \bar{\phi}:=f_{B_{5 R}^{+}\left(x_{1}\right)} \phi
$$

Furthermore, since $\mathcal{S}$ is compact, there is a point $Q \in \mathcal{S} \operatorname{such}$ that $\operatorname{dist}(\bar{\phi}, \mathcal{S})=\operatorname{dist}(\bar{\phi}, Q)$. Hence

$$
\operatorname{dist}\left(\phi\left(x_{0}\right), Q\right) \leq\left|\phi\left(x_{0}\right)-\bar{\phi}\right|+\operatorname{dist}(\bar{\phi}, Q) \leq C \sqrt{\epsilon_{0}} .
$$

The above lemma shows that

$$
\phi\left(B_{1 / 4}^{+}\right) \subset \mathbf{U}_{\delta}:=\left\{z \in N \mid \operatorname{dist}^{N}(z, \mathcal{S})<\delta\right\}
$$

for some $\delta>0$, provided that the energy of $\phi$ over the half-disk is sufficiently small.

Let $(\phi, \psi) \in \mathcal{X}_{1,4 / 3}^{1,2}\left(B_{1}^{+}, N ; \mathcal{S}\right)$ be a weakly Dirac-harmonic map with free boundary on $\mathcal{S}$. By the conformal invariance of weakly Dirac-harmonic maps from surfaces, we can assume that $\phi\left(B_{1}^{+}\right) \subset \mathbf{U}_{\delta}$.

Denote

$$
\Sigma(x):=D \sigma(\phi(x)), \quad x \in B_{1}^{+} .
$$

Define a morphism $\mathbf{T}_{\phi}^{ \pm}: W^{1,4 / 3}\left(\Sigma B_{1}^{+} \otimes \phi^{-1} T N\right) \rightarrow W^{1,4 / 3}\left(\Sigma B_{1}^{+} \otimes(\sigma \circ \phi)^{-1} T N\right)$ by

$$
\mathbf{T}_{\phi}^{ \pm}:= \pm i \gamma_{1} \otimes \Sigma
$$

Here $\mathbf{T}_{\phi}^{ \pm}$corresponds to $\mathbf{B}_{\phi}^{ \pm}$. Below, we will only consider the case of $\left(\mathbf{B}_{\phi}^{+}, \mathbf{T}_{\phi}^{+}\right)$and omit the symbol " + ", because the case of $\left(\mathbf{B}_{\phi}^{-}, \mathbf{T}_{\phi}^{-}\right)$is analogous. 
For $x=\left(x_{1}, x_{2}\right)$, denoting $x^{*}:=\left(x_{1},-x_{2}\right)$, we extend the fields $\phi, \psi$ to the lower half-disk $B_{1}^{-}:=\left\{\left(x_{1}, x_{2}\right) \in \mathbb{R}^{2} \mid x_{1}^{2}+x_{2}^{2}<1, x_{2} \leq 0\right\}$ as follows (and still denote them by $\phi, \psi)$ :

$$
\begin{array}{ll}
\phi\left(x^{*}\right):=\sigma(\phi(x)), & x^{*} \in B_{1}^{-}, \\
\psi\left(x^{*}\right):=\mathbf{T}_{\phi}(x) \psi(x), & x^{*} \in B_{1}^{-} .
\end{array}
$$

This extension is well defined. To see this, we verify that for a.e. $x \in I$,

$\phi(x)=\sigma(\phi(x)), \quad \psi(x)=(-\vec{n} G \otimes R(x)) \psi(x)=\left(i \gamma_{1} \otimes \Sigma(x)\right) \psi(x)=\mathbf{T}_{\phi}(x) \psi(x)$.

Using the extended map $\phi$, we can extend $\Sigma(x)$ to $B_{1}$. Since $\sigma=\sigma^{-1}$, one verifies that (see also [26])

$$
\Sigma^{-1}(x)=D \sigma(\phi(x))^{-1}=D \sigma(\sigma(\phi(x)))=D \sigma\left(\phi\left(x^{*}\right)\right)=\Sigma\left(x^{*}\right),
$$

so $\Sigma(x) \Sigma\left(x^{*}\right)=\operatorname{Id}(\phi(x))$. Moreover, we can extend $\mathbf{T}_{\phi}$ to some morphism (still denoted by $\left.\mathbf{T}_{\phi}\right) W^{1,4 / 3}\left(\Sigma B_{1} \otimes \phi^{-1} T N\right) \rightarrow W^{1,4 / 3}\left(\Sigma B_{1} \otimes(\sigma \circ \phi)^{-1} T N\right)$. Note that for $\psi \in$ $W^{1,4 / 3}\left(\Sigma B_{1} \otimes \phi^{-1} T N\right)$, if we write $\psi(x)=\psi^{i}(x) \otimes V_{i}(x), x \in B_{1}$, then

$$
\psi\left(x^{*}\right)=\mathbf{T}_{\phi}(x) \psi(x)=i \gamma_{1} \psi^{i}(x) \otimes \Sigma(x) V_{i}(x), \quad x^{*} \in B_{1} .
$$

One checks that $\mathbf{T}_{\phi}(x) \mathbf{T}_{\phi}\left(x^{*}\right) \psi\left(x^{*}\right)=\psi\left(x^{*}\right)$ for any $\psi \in W^{1,4 / 3}\left(\Sigma B_{1} \otimes \phi^{-1} T N\right)$.

Remark 3.3. We note that our reflection for Dirac-harmonic maps is a natural generalization of the one for harmonic maps considered by Gulliver-Jost [13] and Scheven [26].

Using the geodesic reflection $\sigma$, we are able to extend the metric on the bundle $\phi^{-1} T N \rightarrow B_{1}^{+}$to some metric $h$ on the bundle $\phi^{-1} T N \rightarrow B_{1}$ with the extended map $\phi$ as follows:

$$
\langle V(x), W(x)\rangle_{h}:= \begin{cases}\langle V(x), W(x)\rangle, & x \in B_{1}^{+}, \\ \langle\Sigma(x) V(x), \Sigma(x) W(x)\rangle, & x \in B_{1}^{-},\end{cases}
$$

where $V, W \in \Gamma\left(B_{1}, \phi^{-1} T N\right)$. Consequently, the induced metrics on $\Sigma B_{1}^{+} \otimes \phi^{-1} T N$, $T B_{1}^{+} \otimes \phi^{-1} T N$ and $T^{*} B_{1}^{+} \otimes \phi^{-1} T N$ extend to metrics (with respect to $h$ ) on $\Sigma B_{1} \otimes$ $\phi^{-1} T N, T B_{1} \otimes \phi^{-1} T N$ and $T^{*} B_{1} \otimes \phi^{-1} T N$.

Lemma 3.2. For $\psi, \varphi \in W^{1,4 / 3}\left(\Sigma B_{1} \otimes \phi^{-1} T N\right)$,

$$
\langle\psi(x), \varphi(x)\rangle_{h}=\left\langle\mathbf{T}_{\phi}(x) \psi(x), \mathbf{T}_{\phi}(x) \varphi(x)\right\rangle, \quad \forall x \in B_{1}^{-} .
$$

Proof. Given $\psi, \varphi \in W^{1,4 / 3}\left(\Sigma B_{1} \otimes \phi^{-1} T N\right)$, we write $\psi(x)=\psi^{i}(x) \otimes V_{i}(x)$ and $\varphi(x)=\varphi^{j}(x) \otimes V_{j}(x)$. Then for $x \in B_{1}^{-}$,

$$
\begin{aligned}
\langle\psi(x), \varphi(x)\rangle_{h} & =\left\langle\psi^{i}(x), \varphi^{j}(x)\right\rangle\left\langle\Sigma(x) V_{i}(x), \Sigma(x) V_{j}(x)\right\rangle \\
& =\left\langle i \gamma_{1} \psi^{i}(x), i \gamma_{1} \varphi^{j}(x)\right\rangle\left\langle\Sigma(x) V_{i}(x), \Sigma(x) V_{j}(x)\right\rangle \\
& =\left\langle\mathbf{T}_{\phi}(x) \psi(x), \mathbf{T}_{\phi}(x) \varphi(x)\right\rangle .
\end{aligned}
$$


Note that given a vector field $V(x) \in T_{\phi(x)} N$ for $x \in B_{1}$, we have $\Sigma(x) V(x)=$ $D \sigma(\phi(x)) V(x) \in T_{\sigma \circ \phi(x)} N$. We define the covariant derivative $\nabla^{h}$ with respect to $h$ as follows (see also [26]):

$$
\begin{array}{rl}
\nabla_{X(x)}^{h} & V(x) \\
& := \begin{cases}\nabla_{\phi_{*}(X(x))} V(x), \\
\Sigma\left(x^{*}\right) \nabla_{(\sigma \circ \phi)_{*}(X(x))}(\Sigma(x) V(x))=\Sigma\left(x^{*}\right) \nabla_{\Sigma(x) \phi_{*}(X(x))}(\Sigma(x) V(x)), & x \in B_{1}^{+},\end{cases}
\end{array}
$$

where $X \in \Gamma\left(T B_{1}\right), V \in \Gamma\left(B_{1}, \phi^{-1} T N\right)$ and $\nabla$ is the Levi-Civita connection on $N$ (also denote the induced connection for $\phi^{-1} T N$ by $\nabla$ ). One easily verifies that $\nabla^{h}$ is compatible with $h$ :

$$
d\left(\langle V(x), W(x)\rangle_{h}\right)=\left\langle\nabla^{h} V(x), W(x)\right\rangle_{h}+\left\langle V(x), \nabla^{h} W(x)\right\rangle_{h}, \quad x \in B_{1} .
$$

Moreover, we define the tensor $R^{h}(\phi)$ (with symmetries similar to the Riemann curvature tensor $R(\phi))$ by

$$
\begin{aligned}
R^{h}(\phi)(V(x), W(x)) U(x) & \\
& := \begin{cases}R(\phi)(V(x), W(x)) U(x), & x \in B_{1}^{+}, \\
\Sigma\left(x^{*}\right) R(\phi)(\Sigma(x) V(x), \Sigma(x) W(x))(\Sigma(x) U(x)), & x \in B_{1}^{-}\end{cases}
\end{aligned}
$$

Recall that the Dirac operator along the map $\phi$ can be written as

$$
\not D=\not \partial \otimes I d+\gamma_{\alpha} \otimes \nabla_{\phi_{*}\left(\gamma_{\alpha}\right)} .
$$

Now we define the Dirac operator along the extended map $\phi$ with respect to the extended metric $h$ as follows:

$$
\not D^{h}:=\not \partial \otimes I d+\gamma_{\alpha} \otimes \nabla_{\gamma_{\alpha}}^{h}
$$

The following lemma gives a relation between $\not D^{h}$ and $\not D$ :

Lemma 3.3. For any $\xi \in W^{1,4 / 3}\left(\Sigma B_{1} \otimes \phi^{-1} T N\right)$, denote $\xi^{*}(x):=\mathbf{T}_{\phi}\left(x^{*}\right) \xi\left(x^{*}\right)$ for $x \in B_{1}$. Then

$$
\not D_{x^{*}}^{h} \xi\left(x^{*}\right)=\mathbf{T}_{\phi}(x) \not D_{x} \xi^{*}(x), \quad \forall x \in B_{1} .
$$

Proof. Write $\xi=\xi^{i} \otimes V_{i}$. Then, for all $x \in B_{1}$,

$$
\begin{aligned}
\mathbf{T}_{\phi}(x) & \not D_{x} \xi^{*}(x)=\mathbf{T}_{\phi}(x) \not D_{x}\left(i \gamma_{1} \xi^{i}\left(x^{*}\right) \otimes \Sigma\left(x^{*}\right) V_{i}\left(x^{*}\right)\right) \\
= & \mathbf{T}_{\phi}(x)\left\{\not \partial_{x}\left(i \gamma_{1} \xi^{i}\left(x^{*}\right)\right) \otimes \Sigma\left(x^{*}\right) V_{i}\left(x^{*}\right)+\gamma_{\alpha}\left(i \gamma_{1}\right) \xi^{i}\left(x^{*}\right) \otimes \nabla_{\phi_{*}\left(\partial x_{\alpha}\right)}\left(\Sigma\left(x^{*}\right) V_{i}\left(x^{*}\right)\right)\right\} \\
= & \left(i \gamma_{1}\right) \not \partial_{x}\left(i \gamma_{1} \xi^{i}\left(x^{*}\right)\right) \otimes \Sigma(x) \Sigma\left(x^{*}\right) V_{i}\left(x^{*}\right) \\
& +\left(i \gamma_{1}\right) \gamma_{\alpha}\left(i \gamma_{1}\right) \xi^{i}\left(x^{*}\right) \otimes \Sigma(x) \nabla_{\phi_{*}\left(\partial x_{\alpha}\right)}\left(\Sigma\left(x^{*}\right) V_{i}\left(x^{*}\right)\right) \\
= & \not x^{*} \xi^{i}\left(x^{*}\right) \otimes V_{i}\left(x^{*}\right)+\gamma_{\alpha} \xi^{i}\left(x^{*}\right) \otimes \Sigma(x) \nabla_{\Sigma\left(x^{*}\right) \phi_{*}\left(\partial x_{\alpha}^{*}\right)}\left(\Sigma\left(x^{*}\right) V_{i}\left(x^{*}\right)\right) .
\end{aligned}
$$


Here, we have used the fact that

$$
\left(i \gamma_{1}\right) \not \partial_{x}\left(i \gamma_{1} \xi^{i}\left(x^{*}\right)\right)=\not \not_{x *} \xi^{i}\left(x^{*}\right)
$$

and the following identities (which can be verified using $\phi(x)=\sigma\left(\phi\left(x^{*}\right)\right)$ ):

$$
\phi_{*}\left(\partial x_{1}\right)=\Sigma\left(x^{*}\right) \phi_{*}\left(\partial x_{1}^{*}\right), \quad \phi_{*}\left(\partial x_{2}\right)=-\Sigma\left(x^{*}\right) \phi_{*}\left(\partial x_{2}^{*}\right) .
$$

On the other hand, by the definition of $\not D^{h}$, we see that for all $x^{*} \in B_{1}$,

$$
\begin{aligned}
& \not D_{x^{*}}^{h} \xi\left(x^{*}\right)=\not \partial x_{x^{*}} \xi^{i}\left(x^{*}\right) \otimes V_{i}\left(x^{*}\right)+\gamma_{\alpha} \xi^{i}\left(x^{*}\right) \otimes \nabla_{\partial x_{\alpha}^{*}}^{h}\left(V_{i}\left(x^{*}\right)\right) \\
& \quad=\not x_{x^{*}} \xi^{i}\left(x^{*}\right) \otimes V_{i}\left(x^{*}\right)+\gamma_{\alpha} \xi^{i}\left(x^{*}\right) \otimes \Sigma(x) \nabla_{\Sigma\left(x^{*}\right) \phi_{*}\left(\partial x_{\alpha}^{*}\right)}\left(\Sigma\left(x^{*}\right) V_{i}\left(x^{*}\right)\right) .
\end{aligned}
$$

Combining (3.46) and (3.47) proves the lemma.

Theorem 3.1. Let $(\phi, \psi) \in \mathcal{X}_{1,4 / 3}^{1,2}\left(B_{1}^{+}, N ; \mathcal{S}\right)$ be a weakly Dirac-harmonic map with free boundary on $\mathcal{S}$. Extend the fields $\phi, \psi$ to the whole disk $B_{1}$ as before. Then

$$
\begin{aligned}
& \int_{B_{1}} d \phi \cdot h \nabla^{h} V+\int_{B_{1}}\left\langle\psi^{i}, \gamma_{\alpha} \cdot \psi^{j}\right\rangle\left\langle V_{i}, R^{h}(\phi)\left(V, \phi_{*}\left(\partial x_{\alpha}\right)\right) V_{j}\right\rangle_{h}=0, \\
& \int_{B_{1}}\left\langle\psi, \not D^{h} \xi\right\rangle_{h}=0
\end{aligned}
$$

for all compactly supported $V \in H^{1} \cap L^{\infty}\left(B_{1}, \phi^{-1} T N\right)$ and all compactly supported $\xi \in W^{1,4 / 3} \cap L^{\infty}\left(\Sigma B_{1} \otimes \phi^{-1} T N\right)$.

Proof. First, given a compactly supported vector field $V \in H^{1} \cap L^{\infty}\left(B_{1}, \phi^{-1} T N\right)$, we proceed as in [26] to decompose the vector field $V$ into the equivariant and the antiequivariant part with respect to the diffeomorphism $\sigma$, namely, $V=V_{e}+V_{a}$, where for $x \in B_{1}$,

$$
V_{e}(x):=\frac{1}{2}\left[V(x)+\Sigma\left(x^{*}\right) V\left(x^{*}\right)\right], \quad V_{a}(x):=\frac{1}{2}\left[V(x)-\Sigma\left(x^{*}\right) V\left(x^{*}\right)\right] .
$$

Since $\Sigma(x) \Sigma\left(x^{*}\right)=\operatorname{Id}(\phi(x))$, one checks that

$$
V_{e}\left(x^{*}\right)=\Sigma(x) V(x), \quad V_{a}\left(x^{*}\right)=-\Sigma(x) V_{a}(x) .
$$

By (3.44), we have, for $x_{0} \in I$,

$$
V_{e}\left(x_{0}\right)=\frac{1}{2}\left[V\left(x_{0}\right)+\Sigma\left(x_{0}\right) V\left(x_{0}\right)\right] \in T_{\phi\left(x_{0}\right)} \mathcal{S} .
$$

Hence, $\left.V_{e}\right|_{B_{1}^{+}}$is an admissible variation vector field for $\phi$ with respect to the free boundary condition $\phi(I) \subset \mathcal{S}$. It follows from Proposition 3.7 that

$$
\int_{B_{1}^{+}} d \phi \cdot \nabla V_{e}+\int_{B_{1}^{+}}\left\langle\psi^{i}, \gamma_{\alpha} \cdot \psi^{j}\right\rangle\left\langle V_{i}, R(\phi)\left(V_{e}, \phi_{*}\left(\partial x_{\alpha}\right)\right) V_{j}\right\rangle=0 .
$$


Applying the equivariance of $V_{e}$ and the symmetry properties of $\nabla^{h}$ (see its definition), one verifies

$$
\int_{B_{1}^{-}} d \phi \cdot h \nabla^{h} V_{e}=\int_{B_{1}^{+}} d \phi \cdot \nabla V_{e}
$$

In view of the antiequivariance of $V_{a}$, we analogously obtain

$$
\int_{B_{1}^{-}} d \phi \cdot h \nabla^{h} V_{a}=-\int_{B_{1}^{+}} d \phi \cdot \nabla V_{a} .
$$

Recall that $\psi\left(x^{*}\right)=\left(i \gamma_{\alpha}\right) \psi^{i}(x) \otimes \Sigma(x) V_{i}(x)$. We claim that the following two identities hold:

$$
\begin{gathered}
\int_{x^{*} \in B_{1}^{-}}\left\langle\left(i \gamma_{1}\right) \psi^{i}(x), \gamma_{\alpha} \cdot\left(i \gamma_{1}\right) \psi^{j}(x)\right\rangle\left\langle\Sigma(x) V_{i}(x), R^{h}(\phi)\left(V_{e}, \phi_{*}\left(\partial x_{\alpha}^{*}\right)\right) \Sigma(x) V_{j}(x)\right\rangle_{h} \\
=\int_{x \in B_{1}^{+}}\left\langle\psi^{i}(x), \gamma_{\alpha} \cdot \psi^{j}(x)\right\rangle\left\langle V_{i}(x), R(\phi)\left(V_{e}(x), \phi_{*}\left(\partial x_{\alpha}\right)\right) V_{j}(x)\right\rangle, \\
\int_{x^{*} \in B_{1}^{-}}\left\langle\left(i \gamma_{1}\right) \psi^{i}(x), \gamma_{\alpha} \cdot\left(i \gamma_{1}\right) \psi^{j}(x)\right\rangle\left\langle\Sigma(x) V_{i}(x), R^{h}(\phi)\left(V_{a}, \phi_{*}\left(\partial x_{\alpha}^{*}\right)\right) \Sigma(x) V_{j}(x)\right\rangle_{h} \\
=-\int_{x \in B_{1}^{+}}\left\langle\psi^{i}(x), \gamma_{\alpha} \cdot \psi^{j}(x)\right\rangle\left\langle V_{i}(x), R(\phi)\left(V_{a}, \phi_{*}\left(\partial x_{\alpha}\right)\right) V_{j}(x)\right\rangle .
\end{gathered}
$$

If the claim is true, then combining (3.48)-(3.52) gives

$$
\int_{B_{1}} d \phi \cdot h \nabla^{h} V+\int_{B_{1}}\left\langle\psi^{i}, \gamma_{\alpha} \cdot \psi^{j}\right\rangle\left\langle V_{i}, R^{h}(\phi)\left(V, \phi_{*}\left(\partial x_{\alpha}\right)\right) V_{j}\right\rangle_{h}=0 .
$$

Now it is sufficient to prove the claim. Let $x=\left(x_{1}, x_{2}\right) \in B_{1}^{+}$; then $x^{*}=\left(x_{1},-x_{2}\right)$ $\in B_{1}^{-}$. Since $\phi\left(x^{*}\right)=\sigma(\phi(x))$, we have

$$
\phi_{*}\left(\partial x_{1}^{*}\right)=\Sigma(x) \phi_{*}\left(\partial x_{1}\right), \quad \phi_{*}\left(\partial x_{2}^{*}\right)=-\Sigma(x) \phi_{*}\left(\partial x_{2}\right) .
$$

Hence,

$$
\begin{aligned}
\left\langle\left(i \gamma_{1}\right) \psi^{i}(x), \gamma_{\alpha} \cdot\left(i \gamma_{1}\right) \psi^{j}(x)\right\rangle\left\langle\Sigma(x) V_{i}(x), R^{h}(\phi)\left(V_{e}\left(x^{*}\right), \phi_{*}\left(\partial x_{\alpha}^{*}\right)\right) \Sigma(x) V_{j}(x)\right\rangle_{h} \\
=\left\langle\left(i \gamma_{1}\right) \psi^{i}(x), \gamma_{1} \cdot\left(i \gamma_{1}\right) \psi^{j}(x)\right\rangle \\
\quad \times\left\langle\Sigma(x) V_{i}(x), R^{h}(\phi)\left(\Sigma(x) V_{e}(x), \Sigma(x) \phi_{*}\left(\partial x_{1}\right)\right) \Sigma(x) V_{j}(x)\right\rangle_{h} \\
+\quad\left\langle\left(i \gamma_{1}\right) \psi^{i}(x), \gamma_{2} \cdot\left(i \gamma_{1}\right) \psi^{j}(x)\right\rangle \\
\quad \times\left\langle\Sigma(x) V_{i}(x), R^{h}(\phi)\left(\Sigma(x) V_{e}(x),-\Sigma(x) \phi_{*}\left(\partial x_{2}\right)\right) \Sigma(x) V_{j}(x)\right\rangle_{h} \\
=\left\langle\psi^{i}(x), \gamma_{\alpha} \cdot \psi^{j}(x)\right\rangle\left\langle\Sigma(x) V_{i}(x), R^{h}(\phi)\left(\Sigma(x) V_{e}(x), \Sigma(x) \phi_{*}\left(\partial x_{\alpha}\right)\right) \Sigma(x) V_{j}(x)\right\rangle_{h} \\
=\left\langle\psi^{i}(x), \gamma_{\alpha} \cdot \psi^{j}(x)\right\rangle\left\langle\Sigma(x) V_{i}(x), \Sigma(x) R(\phi)\left(V_{e}(x), \phi_{*}\left(\partial x_{\alpha}\right)\right) V_{j}(x)\right\rangle_{h} \\
=\left\langle\psi^{i}(x), \gamma_{\alpha} \cdot \psi^{j}(x)\right\rangle\left\langle V_{i}(x), R(\phi)\left(V_{e}(x), \phi_{*}\left(\partial x_{\alpha}\right)\right) V_{j}(x)\right\rangle .
\end{aligned}
$$


Integrating the above identity for $x^{*} \in B_{1}^{-}$and changing variables $x^{*} \mapsto x$, we have (3.51). Similarly, using the fact that $V_{a}\left(x^{*}\right)=-\Sigma(x) V_{a}(x)$, one checks (3.52).

Next, given a compactly supported $\xi \in W^{1,4 / 3} \cap L^{\infty}\left(\Sigma B_{1} \otimes \phi^{-1} T N\right)$, we have (recall that $\vec{n}=-\gamma_{2}$ )

$$
\int_{B_{1}^{+}}\left\langle\psi, \not D^{h} \xi\right\rangle_{h}=\int_{B_{1}^{+}}\langle\not D \psi, \xi\rangle-\int_{I}\left\langle\left(-\gamma_{2}\right) \cdot \psi, \xi\right\rangle .
$$

By Lemmas 3.2 and 3.3,

$$
\begin{aligned}
\int_{x^{*} \in B_{1}^{-}}\left\langle\psi\left(x^{*}\right), \not D_{x^{*}}^{h} \xi\left(x^{*}\right)\right\rangle_{h} & =\int_{x^{*} \in B_{1}^{-}}\left\langle\mathbf{T}_{\phi}\left(x^{*}\right) \psi\left(x^{*}\right), \mathbf{T}_{\phi}\left(x^{*}\right) \not D_{x^{*}}^{h} \xi\left(x^{*}\right)\right\rangle \\
& =\int_{x \in B_{1}^{+}}\left\langle\psi(x), \not D_{x} \xi^{*}(x)\right\rangle \\
& =\int_{x \in B_{1}^{+}}\left\langle\not D \psi(x), \xi^{*}(x)\right\rangle-\int_{I}\left\langle\left(-\gamma_{2}\right) \cdot \psi(x), \xi^{*}(x)\right\rangle .
\end{aligned}
$$

Hence,

$$
\int_{B_{1}}\left\langle\psi, \not D^{h} \xi\right\rangle_{h}=\int_{B_{1}^{+}}\left\langle\not D \psi, \xi+\xi^{*}\right\rangle-\int_{I}\left\langle\left(-\gamma_{2}\right) \cdot \psi, \xi+\xi^{*}\right\rangle
$$

For $x \in I$, one verifies that

$$
\begin{aligned}
\mathbf{B}_{\phi}\left(\xi+\xi^{*}\right)(x) & =\frac{1}{2}\left(I \otimes \mathrm{Id}-i \gamma_{1} \otimes \Sigma\right)\left(\xi+\xi^{*}\right)=\frac{1}{2}\left(I \otimes \mathrm{Id}-i \gamma_{1} \otimes \Sigma\right)\left(\xi+i \gamma_{1} \otimes \Sigma \xi\right) \\
& =\frac{1}{2}\left[(I \otimes \mathrm{Id}) \xi-\left(\left(i \gamma_{1}\right)^{2} \otimes \Sigma^{2}\right) \xi\right]=0 .
\end{aligned}
$$

Therefore, $\xi+\xi^{*}$ satisfies the following chirality boundary condition on $I$ :

$$
\left.\mathbf{B}_{\phi}\left(\xi+\xi^{*}\right)\right|_{I}=0 .
$$

Recall that, by assumption, $\psi$ satisfies the same chirality boundary condition. Hence, by Proposition 3.5,

$$
\int_{I}\left\langle\vec{n} \cdot \psi, \xi+\xi^{*}\right\rangle=\int_{I}\left\langle\left(-\gamma_{2}\right) \cdot \psi, \xi+\xi^{*}\right\rangle=0 .
$$

Noting that $\not D \psi=0$ in $B_{1}^{+}$, we conclude from (3.53) that $\int_{B_{1}}\left\langle\psi, \not D^{h} \xi\right\rangle_{h}=0$.

\section{Continuity of weakly Dirac-harmonic maps at a free boundary}

Starting with the global orthonormal frame $V_{i}(x)=\widehat{V}_{i}(\phi(x)), i=1, \ldots, d$, on $\phi^{-1} T N$, we can apply the Gram-Schmidt orthonormalization procedure to construct an $H^{1}$-tangent frame $e_{i}(x) \in T_{\phi(x)} N$ that is orthonormal with respect to $h$ (see [26]). This construction gives the estimate

$$
\sup _{1 \leq i \leq d}\left|\nabla e_{i}(x)\right| \leq C|d \phi(x)|, \quad x \in B_{1}
$$

where $C=C(\mathcal{S}, N)$ is a constant. 
Define

$$
\mathfrak{R}_{l m}:=\sum_{i, j, \alpha}\left\langle\psi^{i}(x), \gamma_{\alpha} \cdot \psi^{j}(x)\right\rangle\left\langle e_{i}, R^{h}(\phi)\left(e_{l}, e_{m}\right) e_{j}\right\rangle_{h} d x_{\alpha}
$$

Then, by the symmetry properties of $R^{h}(\phi)$, one can verify (similarly to [28]) that $\mathfrak{R}_{l m}=$ $-\mathfrak{R}_{m l}$ and $\overline{\mathfrak{R}_{l m}}=\mathfrak{R}_{l m}$, for $1 \leq l, m \leq d$. Moreover, we get

Proposition 3.8. We have $\mathfrak{R}=\left(\mathfrak{R}_{l m}\right) \in L^{2}\left(B_{1}\right.$, so $\left.(d) \otimes \bigwedge^{1} \mathbb{R}^{2}\right)$.

Using $\mathfrak{R}_{l m}$, we can write

$$
\begin{aligned}
\left\langle\psi^{i}(x)\right. & \left., \gamma_{\alpha} \cdot \psi^{j}(x)\right\rangle\left\langle e_{i}, R^{h}(\phi)\left(e_{l}, \phi_{*}\left(\partial x_{\alpha}\right)\right) e_{j}\right\rangle_{h} \\
& =\left\langle\psi^{i}(x), \gamma_{\alpha} \cdot \psi^{j}(x)\right\rangle\left\langle e_{i}, R^{h}(\phi)\left(e_{l},\left(\phi_{*}\left(\partial x_{\alpha}\right) \cdot_{h} e_{m}\right) e_{m}\right) e_{j}\right\rangle_{h} \\
& =\left(\phi_{*}\left(\partial x_{\alpha}\right) \cdot{ }_{h} e_{m}\right)\left\langle\psi^{i}(x), \gamma_{\alpha} \cdot \psi^{j}(x)\right\rangle\left\langle e_{i}, R^{h}(\phi)\left(e_{l}, e_{m}\right) e_{j}\right\rangle_{h}=\mathfrak{R}_{l m} \cdot\left(d \phi \cdot_{h} e_{m}\right) .
\end{aligned}
$$

Note that here $d \phi=\phi_{*}\left(\partial x_{\alpha}\right) d x_{\alpha}$ and $d \phi \cdot_{h} e_{m}=\left(\phi_{*}\left(\partial x_{\alpha}\right) \cdot_{h} e_{m}\right) d x_{\alpha}$.

Given any $\varphi \in C_{0}^{\infty}\left(B_{1}\right)$, fix $1 \leq i \leq d$ and take $V=\varphi e_{i}$ in Theorem 3.1 to get

$$
\begin{aligned}
0 & =\int_{B_{1}} d \phi \cdot h \nabla^{h}\left(\varphi e_{i}\right)+\int_{B_{1}}\left\langle\psi^{l}(x), \gamma_{\alpha} \cdot \psi^{m}(x)\right\rangle\left\langle e_{l}, R^{h}(\phi)\left(\left(\varphi e_{i}\right), \phi_{*}\left(\partial x_{\alpha}\right)\right) e_{m}\right\rangle_{h} \\
& =\int_{B_{1}}\left(d \phi \cdot_{h} e_{i}\right) d \varphi+\int_{B_{1}}\left(\nabla^{h} e_{i} \cdot h e_{j}\right)\left(d \phi \cdot_{h} e_{i}\right) \varphi+\int_{B_{1}} \Re_{i j} \cdot\left(d \phi \cdot_{h} e_{j}\right) \varphi .
\end{aligned}
$$

Since $\varphi \in C_{0}^{\infty}\left(B_{1}\right)$ is arbitrary, we have

$$
d^{*}\left(d \phi \cdot h e_{i}\right)=\left(\left(\nabla^{h} e_{i} \cdot{ }_{h} e_{j}\right)+\mathfrak{R}_{i j}\right)\left(d \phi \cdot{ }_{h} e_{j}\right) .
$$

Noting that $e_{i}(x) \in T_{\phi(x)} N$ is an $H^{1}$-tangent frame that is orthonormal with respect to $h$ and $\nabla^{h}$ is compatible with $h$, one verifies that $\left(\nabla^{h} e_{i} \cdot{ }_{h} e_{j}\right)$ is antisymmetric with respect to the indices $i$ and $j$. Moreover, we have

Proposition 3.9. We have $\left(\nabla^{h} e_{i} \cdot{ }_{h} e_{j}\right)_{i, j} \in L^{2}\left(B_{1}\right.$, so $\left.(d) \otimes \bigwedge^{1} \mathbb{R}^{2}\right)$.

To proceed, let us recall the Coulomb gauge construction theorem due to Rivière [24] and Rivière-Struwe [25] (we only need to consider the case that the domain is twodimensional and hence we use the norm $L^{2}$ instead of $M^{2,2}$ ).

Lemma 3.4. There exist $\epsilon_{1}>0$ and $C>0$ such that if $\Omega \in L^{2}\left(B_{1}, \operatorname{so}(d) \otimes \bigwedge^{1} \mathbb{R}^{2}\right)$ satisfies

$$
\|\Omega\|_{L^{2}\left(B_{1}\right)} \leq \epsilon_{1},
$$

then there exist $P \in H^{1}\left(B_{1}, \mathrm{SO}(d)\right)$ and $\zeta \in H^{1}\left(B_{1}, \operatorname{so}(d) \otimes \bigwedge^{2} \mathbb{R}^{2}\right)$ such that

$$
\begin{aligned}
P^{-1} d P+P^{-1} \Omega P & =d^{*} \zeta & & \text { in } B_{1}, \\
d \zeta & =0 & & \text { in } B_{1}, \\
\zeta & =0 & & \text { on } \partial B_{1} .
\end{aligned}
$$

Moreover, $\nabla P$ and $\nabla \zeta$ belong to $L^{2}\left(B_{1}\right)$ with

$$
\|\nabla P\|_{L^{2}\left(B_{1}\right)}+\|\nabla \zeta\|_{L^{2}\left(B_{1}\right)} \leq C\|\Omega\|_{L^{2}\left(B_{1}\right)} \leq C \epsilon_{1} .
$$


The above lemma can be applied to study the regularity of weakly Dirac-harmonic maps with free boundary when the two fields are extended to the whole disk.

Lemma 3.5. There exists $\epsilon_{2}>0$ such that if $(\phi, \psi) \in \mathcal{X}_{1,4 / 3}^{1,2}\left(B_{1}^{+}, N ; \mathcal{S}\right)$ is a weakly Dirac-harmonic map with free boundary on $\mathcal{S}$ satisfying

$$
\|d \phi\|_{L^{2}\left(B_{1}^{+}\right)}^{2}+\|\psi\|_{L^{4}\left(B_{1}^{+}\right)}^{4} \leq \epsilon_{2}^{2},
$$

then $\phi \in C^{0, \alpha}\left(B_{1 / 2}^{+}, N\right)$ for any $\alpha \in(0,1)$. Moreover,

$$
[\phi]_{C^{0, \alpha}\left(B_{1 / 2}^{+}\right)} \leq C\|d \phi\|_{L^{2}\left(B_{1}^{+}\right)} .
$$

Remark 3.4. The scheme of proof will be similar to the ones of [25, 28], but we need to present the details here in order to set up our framework for the extended metric $h$.

Proof. First we extend the fields $\phi, \psi$ to the whole disk $B_{1}$ as before. Then, combining Propositions 3.8 and 3.9 gives $\Omega=\left(\Omega_{i j}\right):=\left(\left(\nabla^{h} e_{i} \cdot{ }_{h} e_{j}\right)+\Re_{i j}\right) \in L^{2}\left(B_{1}, \operatorname{so}(d) \otimes \bigwedge^{1} \mathbb{R}^{2}\right)$. Moreover, (3.54) gives

$$
\|\Omega\|_{L^{2}\left(B_{1}\right)} \leq C\left[\|d \phi\|_{L^{2}\left(B_{1}^{+}\right)}+\|\psi\|_{L^{4}\left(B_{1}^{+}\right)}^{2}\right] \leq C \epsilon_{2} \leq \epsilon_{1},
$$

where $\epsilon_{1}>0$ is as in Lemma 3.3 and $\epsilon_{2}>0$ is chosen to be sufficiently small. Hence, Lemma 3.4 yields $P \in H^{1}\left(B_{1}, \mathrm{SO}(d)\right)$ and $\zeta \in H^{1}\left(B_{1}, \operatorname{so}(d) \otimes \bigwedge^{2} \mathbb{R}^{2}\right)$ satisfying

$$
\begin{aligned}
P^{-1} d P+P^{-1} \Omega P & =d^{*} \zeta & & \text { in } B_{1}, \\
d \zeta & =0 & & \text { in } B_{1}, \\
\zeta & =0 & & \text { on } \partial B_{1},
\end{aligned}
$$

and

$$
\|\nabla P\|_{L^{2}\left(B_{1}\right)}+\|\nabla \zeta\|_{L^{2}\left(B_{1}\right)} \leq C\|\Omega\|_{L^{2}\left(B_{1}\right)} \leq C \epsilon_{2}
$$

We write $P=\left(P_{i j}\right), P^{-1}=\left(P_{j i}\right)$, and $\zeta=\left(\zeta_{i j}\right)$. Since $P \in H^{1}\left(B_{1}, \mathrm{SO}(d)\right)$ and hence $P^{-1} P=P^{T} P=I_{d}$, we have $d P^{-1}=-P^{-1} d P P^{-1}$. Using (3.55) and (3.56), we calculate

$$
\begin{aligned}
d^{*}\left[P^{-1}\left(\begin{array}{c}
d \phi \cdot_{h} e_{1} \\
\vdots \\
d \phi \cdot_{h} e_{d}
\end{array}\right)\right] & =\left(d P^{-1} P+P^{-1} \Omega P\right) \cdot P^{-1}\left(\begin{array}{c}
d \phi \cdot_{h} e_{1} \\
\vdots \\
d \phi \cdot_{h} e_{d}
\end{array}\right) \\
& =-d^{*} \zeta \cdot P^{-1}\left(\begin{array}{c}
d \phi \cdot_{h} e_{1} \\
\vdots \\
d \phi \cdot_{h} e_{d}
\end{array}\right)
\end{aligned}
$$

Equivalently, we have

$$
-d^{*}\left(P_{j i}\left(d \phi \cdot_{h} e_{j}\right)\right)=d^{*} \zeta_{i l} \cdot\left(P_{m l}\left(d \phi \cdot_{h} e_{m}\right)\right), \quad i=1, \ldots, d, \quad \text { in } B_{1} .
$$


For any $0<R \leq 1 / 4$, let $B_{R} \subset B_{1 / 2}$ be an arbitrary disk of radius $R$ and $\tau \in$ $C_{0}^{\infty}\left(B_{1 / 2}\right)$ satisfying $0 \leq \tau \leq 1, \tau \equiv 1$ in $B_{R}, \tau \equiv 0$ outside $B_{2 R}$, and $|\nabla \tau| \leq 4 / R$. Denote $\widetilde{\phi}:=\tau\left(\phi-\bar{\phi}_{R}\right)$, where $\bar{\phi}_{R}:=f_{B_{R}} \phi$.

For each $1 \leq i \leq d$, the 1 -form $\sum_{j=1}^{d} P_{j i}\left(d \tilde{\phi} \cdot{ }_{h} e_{j}\right) \in L^{2}\left(\mathbb{R}^{2}, \bigwedge^{1} \mathbb{R}^{2}\right)$, extended by 0 outside of $B_{2 R}$, admits a Hodge-de Rham decomposition of the form

$$
\sum_{j=1}^{d} P_{j i}\left(d \widetilde{\phi} \cdot h e_{j}\right)=d f_{i}+d^{*} g_{i}+h_{i}
$$

where $f_{i} \in H_{0}^{1}\left(B_{R}\right), g_{i} \in H_{0}^{1}\left(B_{R}, \bigwedge^{2} \mathbb{R}^{2}\right)$ is a closed 2-form, i.e., $d g_{i}=0$ in $B_{R}$, and $h_{i} \in L^{2}\left(B_{R}, \bigwedge^{1} \mathbb{R}^{2}\right)$ is a harmonic 1-form (we refer to Iwaniec-Martin [18] for more details on the Hodge decomposition of forms in Sobolev spaces).

Taking first $d^{*}$ and then $d$ of both sides of (3.58) and applying (3.57) gives in $B_{R}$, for $1 \leq i \leq d$,

$$
-\Delta f_{i}=d^{*} \zeta_{i l}\left(P_{j l}\left(d \phi{ }_{h} e_{j}\right)\right), \quad \Delta g_{i}=d P_{j i} \wedge\left(d \phi \cdot{ }_{h} e_{j}\right)+P_{j i} d \phi \wedge_{h} d e_{j} .
$$

For $1<p<2$, let $q=p /(p-1)$ be the conjugate exponent. By the duality characterization of $\|\nabla f\|_{L^{p}\left(B_{R}\right)}$ for $f \in W_{0}^{1, p}\left(B_{R}\right)$, we get

$$
\|\nabla f\|_{L^{p}\left(B_{R}\right)} \leq C \sup \left\{\int_{B_{R}} \nabla f \cdot \nabla \varphi d x \mid \varphi \in W_{0}^{1, q}\left(B_{R}\right),\|\nabla \varphi\|_{L^{q}\left(B_{R}\right)} \leq 1\right\} .
$$

Since $q>2$, by the Sobolev embedding theorem, we have $W_{0}^{1, q}\left(B_{R}\right) \hookrightarrow C^{0,1-2 / q}\left(B_{R}\right)$, and for $\varphi \in W_{0}^{1, q}\left(B_{R}\right)$ with $\|\nabla \varphi\|_{L^{q}\left(B_{R}\right)} \leq 1$ the following estimates hold:

$$
\|\varphi\|_{L^{\infty}\left(B_{R}\right)} \leq C R^{1-2 / q}, \quad\|\nabla \varphi\|_{L^{2}\left(B_{R}\right)} \leq C R^{1-2 / q} .
$$

For any such $\varphi$, we can estimate $f_{i}$ (similarly to Rivière-Struwe [25] and Wang-Xu [28]) as follows:

$$
\begin{aligned}
\int_{B_{R}} d f_{i} \cdot d \varphi= & -\int_{B_{R}} \Delta f_{i} \cdot \varphi=\int_{B_{R}} d^{*} \zeta_{i l} \cdot\left(P_{j l}\left(d \phi h_{h} e_{j}\right)\right) \cdot \varphi \\
= & \int_{B_{R}} d^{*} \zeta_{i l} \cdot\left(P_{j l}\left(d \phi \cdot \hat{e}_{j}\right)\right) \cdot \varphi=-\int_{B_{R}} d^{*} \zeta_{i l} \cdot d\left(P_{j l} \hat{e}_{j} \varphi\right) \tilde{\phi} \\
\leq & C\left\|d^{*} \zeta_{i l} \cdot d\left(P_{j l} \hat{e}_{j} \varphi\right)\right\|_{\mathcal{H}^{1}\left(\mathbb{R}^{2}\right)}[\phi]_{\mathrm{BMO}\left(B_{R}\right)} \\
\leq & C\|\nabla \zeta\|_{L^{2}\left(B_{R}\right)}\left(\|\nabla P\|_{L^{2}\left(B_{R}\right)}+\sum_{j}\left\|\nabla \hat{e}_{j}\right\|_{L^{2}\left(B_{R}\right)}\right)\|\varphi\|_{L^{\infty}\left(B_{R}\right)}[\phi]_{\mathrm{BMO}\left(B_{R}\right)} \\
& +C\|\nabla \zeta\|_{L^{2}\left(B_{R}\right)}\|\nabla \varphi\|_{L^{2}\left(B_{R}\right)}[\phi]_{\mathrm{BMO}\left(B_{R}\right)} \\
\leq & C\|\nabla \zeta\|_{L^{2}\left(B_{R}\right)}\left(\|\nabla P\|_{L^{2}\left(B_{R}\right)}+\|d \phi\|_{L^{2}\left(B_{R}\right)}\right)\|\varphi\|_{L^{\infty}\left(B_{R}\right)}[\phi]_{\mathrm{BMO}\left(B_{R}\right)} \\
& +C\|\nabla \zeta\|_{L^{2}\left(B_{R}\right)}\|\nabla \varphi\|_{L^{2}\left(B_{R}\right)}[\phi]_{\mathrm{BMO}\left(B_{R}\right)} \\
\leq & C \epsilon_{2}\left[\|\varphi\|_{L^{\infty}\left(B_{R}\right)}+\|\nabla \varphi\|_{L^{2}\left(B_{R}\right)}\right][\phi]_{\mathrm{BMO}\left(B_{R}\right)} \\
\leq & C \epsilon_{2} R^{2 / p-1}[\phi]_{\mathrm{BMO}\left(B_{R}\right)}
\end{aligned}
$$


where we have used the notations $d \phi \cdot h e_{j}=d \phi \cdot\left(h_{j l} e_{l}\right), \hat{e}_{j}:=h_{j l} e_{l}$, (3.54) and the estimates

$$
\sum_{j}\left|\hat{e}_{j}\right| \leq C \sum_{j}\left|e_{j}\right|, \quad \sum_{j}\left\|\nabla \hat{e}_{j}\right\|_{L^{2}\left(B_{R}\right)} \leq C\|d \phi\|_{L^{2}\left(B_{R}\right)} .
$$

By (3.59), we get

$$
\left(R^{p-2} \int_{B_{R}}\left|\nabla f_{i}\right|^{p}\right)^{1 / p} \leq C \epsilon_{2}[\phi]_{\mathrm{BMO}\left(B_{R}\right)} .
$$

Similarly, for any $\varphi \in W_{0}^{1, q}\left(B_{R}\right)$ satisfying (3.60), we can estimate $g_{i}$ as follows

$$
\begin{array}{rl}
\int_{B_{R}} & d g_{i} \cdot d \varphi=-\int_{B_{R}} \Delta g_{i} \cdot \varphi=-\int_{B_{R}}\left[d P_{j i} \wedge\left(d \phi \cdot_{h} e_{j}\right)+P_{j i} d \phi \wedge_{h} d e_{j}\right] \varphi \\
= & -\int_{B_{R}}\left[d P_{j i} \wedge\left(d \phi \cdot \hat{e}_{j}\right)+P_{j i} d \phi \wedge\left(h_{j l} d e_{l}\right)\right] \varphi \\
= & \int_{B_{R}}\left[d P_{j i} \wedge d\left(\varphi \hat{e}_{j}\right)+d\left(P_{j i} h_{j l} \varphi\right) \wedge d e_{l}\right] \widetilde{\phi} \\
\leq & C\left[\left\|d P_{j i} \wedge d\left(\varphi \hat{e}_{j}\right)\right\|_{\mathcal{H}^{1}\left(\mathbb{R}^{2}\right)}+\left\|d\left(P_{j i} h_{j l} \varphi\right) \wedge d e_{l}\right\|_{\mathcal{H}^{1}\left(\mathbb{R}^{2}\right)}\right][\phi]_{\mathrm{BMO}\left(B_{R}\right)} \\
\leq & C\|\nabla P\|_{L^{2}\left(B_{R}\right)}\left(\|\nabla \varphi\|_{L^{2}\left(B_{R}\right)}+\sum_{j}\left\|\nabla \hat{e}_{j}\right\|_{L^{2}\left(B_{R}\right)}\|\varphi\|_{L^{\infty}\left(B_{R}\right)}\right)[\phi]_{\mathrm{BMO}\left(B_{R}\right)} \\
& +C\left(\sum_{l}\left\|\nabla e_{l}\right\|_{L^{2}\left(B_{R}\right)}\right) \\
& \times\left(\|\nabla \varphi\|_{L^{2}\left(B_{R}\right)}+\left(\|\nabla P\|_{L^{2}\left(B_{R}\right)}+\|\nabla h\|_{L^{2}\left(B_{R}\right)}\right)\|\varphi\|_{L^{\infty}\left(B_{R}\right)}\right)[\phi]_{\mathrm{BMO}\left(B_{R}\right)} \\
\leq & C\|\nabla P\|_{L^{2}\left(B_{R}\right)}\left(\|\nabla \varphi\|_{L^{2}\left(B_{R}\right)}+\|d \phi\|_{L^{2}\left(B_{R}\right)}\|\varphi\|_{L^{\infty}\left(B_{R}\right)}\right)[\phi]_{\mathrm{BMO}\left(B_{R}\right)} \\
& +C\|d \phi\|_{L^{2}\left(B_{R}\right)}\left(\|\nabla \varphi\|_{L^{2}\left(B_{R}\right)}+\|\nabla P\|_{L^{2}\left(B_{R}\right)}\|\varphi\|_{L^{\infty}\left(B_{R}\right)}+\|\varphi\|_{L^{\infty}\left(B_{R}\right)}\right)[\phi]_{\mathrm{BMO}\left(B_{R}\right)} \\
\leq & C \epsilon_{2} R^{2 / p-1}[\phi]_{\mathrm{BMO}\left(B_{R}\right)} .
\end{array}
$$

Again, using (3.59), we have

$$
\left(R^{p-2} \int_{B_{R}}\left|\nabla g_{i}\right|^{p}\right)^{1 / p} \leq C \epsilon_{2}[\phi]_{\mathrm{BMO}\left(B_{R}\right)}
$$

To estimate the harmonic 1-form $h_{i}$, we apply the classical Campanato estimates for harmonic functions (see Giaquinta [11]), (3.61) and (3.62) to get, for any $0<r<R$,

$$
\begin{aligned}
r^{p-2} \int_{B_{r}}\left|h_{i}\right|^{p} & \leq C\left(\frac{r}{R}\right)^{p}\left(R^{p-2} \int_{B_{R}}\left|h_{i}\right|^{p}\right) \\
& \leq C\left(\frac{r}{R}\right)^{p}\left(R^{p-2} \int_{B_{R}}\left|P_{j i}\left(d \widetilde{\phi} \cdot h e_{j}\right)-d f_{i}-d^{*} g_{i}\right|^{p}\right) \\
& \leq C\left(\frac{r}{R}\right)^{p}\left(R^{p-2} \int_{B_{R}}\left(|d \phi|^{p}+\left|\nabla f_{i}\right|^{p}+\left|\nabla g_{i}\right|^{p}\right)\right) \\
& \leq C\left(\frac{r}{R}\right)^{p}\left(R^{p-2} \int_{B_{R}}|d \phi|^{p}+\epsilon_{2}^{p}[\phi]_{\mathrm{BMO}\left(B_{R}\right)}^{p}\right) .
\end{aligned}
$$


To proceed, we note that by the definition of the extended metric $h$, we have (we may need to take $\delta>0$ small enough so that the tubular neighborhood $\mathbf{U}_{\delta}$ is sufficiently close to $\mathcal{S})$

$$
|d \phi| \leq C(N, \mathcal{S}) \sum_{i}\left|d \phi \cdot h e_{i}\right|
$$

Then using $d \tilde{\phi} \cdot{ }_{h} e_{j}=P_{i j}\left(d f_{i}+d^{*} g_{i}+h_{i}\right)$ and $P \in H^{1}\left(B_{1}, \operatorname{SO}(d)\right)$, we can estimate

$$
\begin{aligned}
& r^{p-2} \int_{B_{r}}|d \phi|^{p} \leq C r^{p-2} \int_{B_{R}}\left(\left|\nabla f_{i}\right|^{p}+\left|\nabla g_{i}\right|^{p}\right)+C r^{p-2} \int_{B_{r}}\left|h_{i}\right|^{p} \\
& \leq C r^{p-2} \int_{B_{R}}\left(\left|\nabla f_{i}\right|^{p}+\left|\nabla g_{i}\right|^{p}\right)+C\left(\frac{r}{R}\right)^{p}\left(R^{p-2} \int_{B_{R}}|d \phi|^{p}+\epsilon_{2}^{p}[\phi]_{\mathrm{BMO}\left(B_{R}\right)}^{p}\right) \\
& \leq C\left(\frac{r}{R}\right)^{p}\left\{R^{p-2} \int_{B_{R}}|d \phi|^{p}+\left(\frac{r}{R}\right)^{-2} \epsilon_{2}^{p}[\phi]_{\mathrm{BMO}\left(B_{R}\right)}^{p}\right\} .
\end{aligned}
$$

An iteration argument (see [25, 28] for more details), combined with Morrey's decay lemma (see [11]), implies that $\phi \in C^{0, \alpha}\left(B_{1 / 2}\right)$ for any $\alpha \in(0,1)$, and $[\phi]_{C^{0, \alpha}\left(B_{1 / 2}\right)} \leq$ $C\|d \phi\|_{L^{2}\left(B_{1}\right)}$. Since $\phi$ is extended to $B_{1}$ by reflection, it follows that $[\phi]_{C^{0, \alpha}\left(B_{1 / 2}^{+}\right)} \leq$ $C\|d \phi\|_{L^{2}\left(B_{1}\right)} \leq C\|d \phi\|_{L^{2}\left(B_{1}^{+}\right)}$.

Theorem 3.2. Let $M$ be a compact Riemann spin surface with boundary $\partial M, N$ be any compact Riemannian manifold, and $\mathcal{S}$ be a closed submanifold of $N$. Let $(\phi, \psi)$ be a weakly Dirac-harmonic map from $M$ to $N$ with free boundary on $\mathcal{S}$. Then $\phi \in$ $C^{0, \alpha}(M, N)$ for any $\alpha \in(0,1)$.

Proof. Apply Lemma 3.5 and rescale the fields $\phi, \psi$ if necessary.

Higher regularity of continuous weakly Dirac-harmonic maps at a free boundary

Let $(\phi, \psi)$ be a weakly Dirac-harmonic map from $M$ to $N$ with free boundary on $\mathcal{S} \subset N$ and suppose that $\phi \in C^{0, \alpha}(M, N)$ for any $\alpha \in(0,1)$. For simplicity, we assume that $M=B_{1}^{+}$and consider the higher regularity of $\phi$ at the boundary point $0 \in I$. As before, we take the adapted coordinates $\left\{y^{i}\right\}_{i=1, \ldots, d}$ in some neighborhood $\mathrm{U} \subset \mathbf{U}_{\delta}$ of the point $\phi(0) \in \mathcal{S}$. By conformal invariance and continuity of $\phi$, we can assume that $\phi\left(B_{1}^{+}\right) \subset$ $\mathrm{U} \subset \mathbf{U}_{\delta}$. Denote

$$
\eta_{i}:= \begin{cases}1, & i=1, \ldots, p, \\ -1, & i=p+1, \ldots, d .\end{cases}
$$

Then the extended fields $\phi, \psi$ can be written as follows for $k=1, \ldots, d$ :

$$
\phi^{k}(x)= \begin{cases}\phi^{k}(x), & x \in B_{1}^{+}, \\ \eta_{k} \phi^{k}\left(x^{*}\right), & x \in B_{1}^{-},\end{cases}
$$


and

$$
\psi_{+}^{k}(x)=\left\{\begin{array}{ll}
\psi_{+}^{k}(x), & x \in B_{1}^{+}, \\
\eta_{k} \psi_{-}^{k}\left(x^{*}\right), & x \in B_{1}^{-},
\end{array} \quad \psi_{-}^{k}(x)= \begin{cases}\psi_{-}^{k}(x), & x \in B_{1}^{+}, \\
\eta_{k} \psi_{+}^{k}\left(x^{*}\right), & x \in B_{1}^{-}\end{cases}\right.
$$

One can verify that

$$
\begin{aligned}
\partial y^{k}\left(\phi\left(x^{*}\right)\right) & =\eta_{k} D \sigma(\phi(x)) \partial y^{k}(\phi(x)) \\
& =\eta_{k} \Sigma(x) \partial y^{k}(\phi(x)), \quad x \in B_{1}, \quad k=1, \ldots, d .
\end{aligned}
$$

For convenience, we shall henceforth also denote the extended metric $h$ by $\widetilde{g}$.

Now we define some geometric data associated to the extended metric $\widetilde{g}$, for $x \in B_{1}$ :

$$
\begin{aligned}
\widetilde{g}_{i j}(\phi(x)) & :=\left\langle\partial y^{i}(\phi(x)), \partial y^{j}(\phi(x))\right\rangle_{\tilde{g}}, \\
\widetilde{\Gamma}_{i j}^{k}(\phi(x)) & := \begin{cases}\widetilde{g}^{k l}(\phi(x))\left\langle\nabla_{\partial y^{i}(\phi(x))} \partial y^{j}(\phi(x)), \partial y^{l}(\phi(x))\right\rangle_{\tilde{g}}, & x \in B_{1}^{+}, \\
\widetilde{g}^{k l}(\phi(x))\left\langle\Sigma\left(x^{*}\right) \nabla_{\Sigma(x) \partial y^{i}(\phi(x))} \Sigma(x) \partial y^{j}(\phi(x)), \partial y^{l}(\phi(x))\right\rangle_{\tilde{g}}, & x \in B_{1}^{-},\end{cases} \\
\widetilde{R}_{m l i j}(\phi(x)) & :=\left\langle\partial y^{j}(\phi(x)), R^{h}(\phi)\left(\partial y^{m}(\phi(x)), \partial y^{l}(\phi(x))\right) \partial y^{i}(\phi(x))\right\rangle_{\tilde{g}}, \\
\widetilde{R}_{l i j}^{m}(\phi(x)) & :=\widetilde{g}^{m k}(\phi(x)) \widetilde{R}_{i j l k}(\phi(x)),
\end{aligned}
$$

where $\left(\widetilde{g}^{m k}(\phi(x))\right)_{m k}$ is the inverse matrix of $\left(\widetilde{g}_{m k}(\phi(x))\right)_{m k}$. Then we have

\section{Lemma 3.6.}

$$
\begin{aligned}
& \tilde{g}_{i j}(\phi(x))= \begin{cases}g_{i j}(\phi(x)), & x \in B_{1}^{+}, \\
\eta_{i} \eta_{j} g_{i j}\left(\phi\left(x^{*}\right)\right), & x \in B_{1}^{-},\end{cases} \\
& \widetilde{\Gamma}_{i j}^{k}(\phi(x))= \begin{cases}\Gamma_{i j}^{k}(\phi(x)), & x \in B_{1}^{+}, \\
\eta_{i} \eta_{j} \eta_{k} \Gamma_{i j}^{k}\left(\phi\left(x^{*}\right)\right), & x \in B_{1}^{-},\end{cases} \\
& \widetilde{R}_{m l i j}(\phi(x))= \begin{cases}R_{m l i j}(\phi(x)), & x \in B_{1}^{+}, \\
\eta_{i} \eta_{j} \eta_{l} \eta_{m} R_{m l i j}\left(\phi\left(x^{*}\right)\right), & x \in B_{1}^{-},\end{cases} \\
& \widetilde{R}_{l i j}^{m}(\phi(x))= \begin{cases}R_{l i j}^{m}(\phi(x)), & x \in B_{1}^{+}, \\
\eta_{i} \eta_{j} \eta_{l} \eta_{m} \widetilde{R}_{l i j}^{m}\left(\phi\left(x^{*}\right)\right), & x \in B_{1}^{-} .\end{cases}
\end{aligned}
$$

Proof. By definition of $h$ and $R^{h}(\phi)$, it is sufficient to consider the case of $x \in B_{1}^{-}$. For such $x$,

$$
\begin{aligned}
\widetilde{g}_{i j}(\phi(x)) & =\left\langle\partial y^{i}(\phi(x)), \partial y^{j}(\phi(x))\right\rangle_{\tilde{g}}=\left\langle\Sigma(x) \partial y^{i}(\phi(x)), \Sigma(x) \partial y^{j}(\phi(x))\right\rangle \\
& =\left\langle\eta_{i} \partial y^{i}\left(\phi\left(x^{*}\right)\right), \eta_{j} \partial y^{j}(\phi(x))\right\rangle=\eta_{i} \eta_{j} g_{i j}\left(\phi\left(x^{*}\right)\right) .
\end{aligned}
$$

It is easy to verify that $\widetilde{g}^{i j}(\phi(x))=\eta_{i} \eta_{j} g^{i j}\left(\phi\left(x^{*}\right)\right)$. Moreover,

$$
\begin{aligned}
\widetilde{\Gamma}_{i j}^{k}(\phi(x)) & =\widetilde{g}^{k l}(\phi(x))\left\langle\Sigma(x) \Sigma\left(x^{*}\right) \nabla_{\Sigma(x) \partial y^{i}(\phi(x))} \Sigma(x) \partial y^{j}(\phi(x)), \Sigma(x) \partial y^{l}(\phi(x))\right\rangle \\
& =\eta_{k} \eta_{l} g^{k l}\left(\phi\left(x^{*}\right)\right) \eta_{i} \eta_{j} \eta_{l}\left\langle\nabla_{\partial y^{i}\left(\phi\left(x^{*}\right)\right)} \partial y^{j}\left(\phi\left(x^{*}\right)\right), \partial y^{l}\left(\phi\left(x^{*}\right)\right)\right\rangle \\
& =\eta_{i} \eta_{j} \eta_{k} \Gamma_{i j}^{k}\left(\phi\left(x^{*}\right)\right),
\end{aligned}
$$


$\widetilde{R}_{m l i j}(\phi(x))=\left\langle\partial y^{j}(\phi(x)), R^{h}(\phi)\left(\partial y^{m}(\phi(x)), \partial y^{l}(\phi(x))\right) \partial y^{i}(\phi(x))\right\rangle_{\widetilde{g}}$

$=\left\langle\Sigma(x) \partial y^{j}(\phi(x)), \Sigma(x) \Sigma\left(x^{*}\right) R(\phi)\left(\Sigma(x) \partial y^{m}(\phi(x)), \Sigma(x) \partial y^{l}(\phi(x))\right) \Sigma(x) \partial y^{i}(\phi(x))\right\rangle$

$=\left\langle\eta_{j} \partial y^{j}\left(\phi\left(x^{*}\right)\right), R(\phi)\left(\eta_{m} \partial y^{m}\left(\phi\left(x^{*}\right)\right), \eta_{l} \partial y^{l}\left(\phi\left(x^{*}\right)\right)\right) \eta_{i} \partial y^{i}\left(\phi\left(x^{*}\right)\right)\right\rangle$

$=\eta_{i} \eta_{j} \eta_{l} \eta_{m} R_{m l i j}\left(\phi\left(x^{*}\right)\right)$,

and

$$
\begin{aligned}
\widetilde{R}_{l i j}^{m}(\phi(x)) & =\widetilde{g}^{m k}(\phi(x)) \widetilde{R}_{i j l k}(\phi(x))=\eta_{m} \eta_{k} \eta_{k} \eta_{l} \eta_{i} \eta_{j} g^{m k}\left(\phi\left(x^{*}\right)\right) R_{i j l k}\left(\phi\left(x^{*}\right)\right) \\
& =\eta_{i} \eta_{j} \eta_{l} \eta_{m} R_{l i j}^{m}\left(\phi\left(x^{*}\right)\right) .
\end{aligned}
$$

Remark 3.5. In the adapted coordinates $\left\{y^{i}\right\}$, we have $g_{i j}(y)=0$, for $y \in \mathrm{U}, i \in$ $\{1, \ldots, p\}, j \in\{p+1, \ldots, d\}$. Hence, both $\widetilde{g}_{i j}(\phi)$ and $\widetilde{g}^{i j}(\phi)$ are continuous (they are in fact Lipschitz; see also [13]).

Now we can write the equations of the extended fields $\phi, \psi$ in terms of the data $\tilde{g}_{i j}$, $\widetilde{\Gamma}_{i j}^{k}$ and $\widetilde{R}_{l i j}^{m}$.

Proposition 3.10. With the assumptions and notations as before, the extended fields $\phi, \psi$ satisfy, in $B_{1}$,

$$
\begin{aligned}
\Delta \phi^{m}+\widetilde{\Gamma}_{i j}^{m}(\phi) \phi_{\alpha}^{i} \phi_{\alpha}^{j}-\frac{1}{2} \widetilde{R}_{l i j}^{m}(\phi)\left\langle\psi^{i}, \nabla \phi^{l} \cdot \psi^{j}\right\rangle & =0, \quad m=1, \ldots, d, \\
\not \partial \psi^{i}+\widetilde{\Gamma}_{j k}^{i}(\phi) \partial_{\alpha} \phi^{j} \gamma_{\alpha} \cdot \psi^{k} & =0, \quad i=1, \ldots, d,
\end{aligned}
$$

Proof. Noting that $\phi\left(B_{1}^{+}\right) \subset \mathrm{U}$, the proposition follows by applying Lemma 3.6 and Theorem 3.1 with $V_{i}(x)=\partial y^{i}(\phi(x)), V(x)=\widetilde{g}^{m j}(\phi(x)) \eta_{j}(x) \otimes \partial y^{m}(\phi(x)), \xi=$ $\widetilde{g}^{m k}(\phi(x)) \xi_{k}(x) \otimes \partial y^{m}(\phi(x))$, where $\eta_{j} \in H_{0}^{1} \cap L^{\infty}\left(B_{1}\right)$ and $\xi_{k} \in W_{0}^{1,4 / 3} \cap L^{\infty}\left(\Sigma B_{1}\right)$ are arbitrarily chosen.

Proposition 3.11. With the assumptions and notations as before, if in addition we assume that $\mathcal{S}$ is totally geodesic, then for all $m, i, j \in\{1, \ldots, d\}$ and any $\gamma \in(0,1)$,

$$
\widetilde{\Gamma}_{i j}^{m}(\phi) \in C^{0, \gamma}\left(B_{1}\right) .
$$

Proof. By definition, we have $\widetilde{\Gamma}_{i j}^{m}(\phi(x))=\eta_{i} \eta_{j} \eta_{m} \Gamma_{i j}^{m}\left(\phi\left(x^{*}\right)\right)$ for $x \in B_{1}^{+}$. Note that both $\Gamma_{i j}^{m}$ and $\phi$ are continuous, hence, to prove the continuity of $\widetilde{\Gamma}_{i j}^{m}(\phi)$, it is sufficient to show that the terms

$$
\Gamma_{\mathrm{T}}^{\perp}, \quad \Gamma_{\mathrm{T}}^{\top}, \quad \Gamma_{\perp \perp}^{\perp}
$$

vanish on $\mathcal{S}$. Here and below, $T$ denotes the tangential index $\{1, \ldots, p\}$ and $\perp$ denotes the normal index $\{p+1, \ldots, d\}$. To verify this, firstly we note that (see [12])

$$
g_{\perp \perp} \equiv 1 \quad \text { on } U, \quad g_{\top \perp} \equiv 0 \quad \text { on } U .
$$

It follows that

$$
g_{\perp \perp, \perp}=g_{\perp \perp, \top}=g_{\top \perp, \perp}=g_{\top \perp, \top} \equiv 0 \quad \text { on } U .
$$


Next, we calculate

$$
\begin{array}{ll}
\Gamma_{\top \top}^{\perp}=\frac{1}{2} g^{\perp \perp}\left(g_{\perp \top, \top}+g_{\perp \top, \top}-g_{\top \top, \perp}\right)=-\frac{1}{2} g^{\perp \perp} g_{\top \top, \perp} & \text { on } U, \\
\Gamma_{\top \perp}^{\top}=\frac{1}{2} g^{\top \top}\left(g_{\top \perp, \top}+g_{\top \top, \perp}-g_{\top \perp, \top}\right)=\frac{1}{2} g^{\top \top} g_{\top \top, \perp} & \text { on } U, \\
\Gamma_{\perp \perp}^{\perp}=\frac{1}{2} g^{\perp \perp} g_{\perp \perp, \perp}=0 \quad \text { on } U . &
\end{array}
$$

Since $\mathcal{S}$ is totally geodesic, we have $\Gamma_{\top}^{\perp}{ }_{\top}=0$ on $\mathcal{S}$. Therefore,

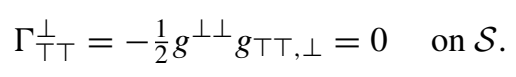

By (3.64), it follows that

$$
\Gamma_{\top \perp}^{\top}=\frac{1}{2} g^{\top \top} g_{\top \top, \perp}=0 \quad \text { on } \mathcal{S} .
$$

Now we have verified that all the terms in (3.63) vanish on $\mathcal{S}$ and hence $\widetilde{\Gamma}_{i j}^{m}(\phi) \in C^{0}$. Moreover, we can write

$$
\widetilde{\Gamma}_{i j}^{m}(\phi(x))= \begin{cases}\Gamma_{i j}^{m}(\phi(x)), & x \in B_{1}^{+}, \\ \Gamma_{i j}^{m}\left(\phi\left(x^{*}\right)\right), & x \in B_{1}^{-} .\end{cases}
$$

Note that $\phi\left(B_{1}^{+}\right) \subset \mathrm{U}, \Gamma_{i j}^{m} \in C^{1}(\overline{\mathrm{U}})$ and $\phi \in C^{0, \gamma}\left(B_{1}^{+}\right)$for any $\gamma \in(0,1)$. Therefore, for any $\gamma \in(0,1)$, we have $\left\|\widetilde{\Gamma}_{i j}^{m}(\phi)\right\|_{C^{0, \gamma}\left(B_{1}\right)} \leq 2\left\|\Gamma_{i j}^{m}(\phi)\right\|_{C^{0, \gamma}\left(B_{1}^{+}\right)}<\infty$.

Theorem 3.3. Let $M$ be a compact Riemann spin surface with boundary $\partial M, N$ be any compact Riemannian manifold, and $\mathcal{S}$ be a closed, totally geodesic submanifold of $N$. Let $(\phi, \psi)$ be a weakly Dirac-harmonic map from $M$ to $N$ with free boundary on $\mathcal{S}$ and suppose that $\phi \in C^{0, \alpha}(M, N)$ for any $\alpha \in(0,1)$. Then there exists $\beta \in(0,1)$ such that $\phi \in C^{1, \beta}(M, N)$ and $\psi \in C^{1, \beta}\left(\Sigma M \otimes \phi^{-1} T N\right)$.

Proof. Combining Lemma 3.6, Proposition 3.10, Proposition 3.11 and applying arguments similar to the proof of [7, Theorem 2.3], we get $\phi \in C^{1, \beta}(M, N)$ and $\psi \in$ $C^{1, \beta}\left(\Sigma M \otimes \phi^{-1} T N\right)$ for some $\beta \in(0,1)$.

Remark 3.6. Following the same strategy as in the proof of [7, Theorem 2.3], take $G=$ $\left(G^{1}, \ldots, G^{d}\right)$, where

$$
G^{m}(x, \phi, d \phi):=\widetilde{\Gamma}_{i j}^{m}(\phi) \phi_{\alpha}^{i} \phi_{\alpha}^{j}-\frac{1}{2} \widetilde{R}_{l i j}^{m}(\phi)\left\langle\psi^{i}, \nabla \phi^{l} \cdot \psi^{j}\right\rangle .
$$

Then using the formulas Lemma 3.6, we have the following pointwise estimate (used in [7, (2.41), p. 70]):

$$
|\nabla G| \leq C(N, \mathcal{S})\left(|d \phi|^{3}+|\psi||\nabla \psi||d \phi|+|\psi|^{2}|d \phi|^{2}+\left|\nabla^{2} \phi\right||d \phi|+\left|\nabla^{2} \phi\right||\psi|^{2}\right)
$$

a.e. in $B_{1}$.

\section{Dirichlet boundary problem for Dirac-harmonic maps}

In this section, we shall study the Dirichlet boundary problem for weakly Dirac-harmonic maps. 
To proceed, we recall that the regularity up to the boundary for weak solutions satisfying (2.21) with continuous boundary trace was established by Müller-Schikorra [22]. More precisely, they proved

Theorem C. Let $D \subset \mathbb{R}^{2}$ be a simply connected domain with $C^{2}$ boundary $\partial D$. Let $u \in H^{1}\left(D, \mathbb{R}^{K}\right)$ and $f \in L^{s}\left(D, \mathbb{R}^{K}\right), s>1$, satisfy

$$
-\Delta u=\Omega \cdot \nabla u+f,\left.\quad u\right|_{\partial D} \in C^{0},
$$

where $\Omega=\left(\Omega_{j}^{i}\right)_{1 \leq i, j \leq K} \in L^{2}\left(D, \operatorname{so}(K) \otimes \mathbb{R}^{2}\right)$. Then $u$ is continuous up to the boundary.

In view of the extrinsic equation (2.26) in the proof of Theorem 2.1, we can apply Theorem $\mathrm{C}$ to obtain the following Dirichlet boundary regularity for weakly Dirac-harmonic maps:

Theorem 4.1. Let $(\phi, \psi)$ be a weakly Dirac-harmonic map from $B_{1}$ to a compact Riemannian manifold $N$. If $\phi$ satisfies the Dirichlet boundary value condition $\left.\phi\right|_{\partial B_{1}} \in C^{0}$, then $\phi$ is continuous up to the boundary.

Proof. We proceed as in the proof of Theorem 2.1. Recall that the equations for the map $\phi$ can be written in the form

$$
-\Delta \phi^{m}=\Omega_{i}^{m} \cdot \nabla \phi^{i}
$$

with some $\Omega=\left(\Omega_{i}^{m}\right)_{1 \leq i, m \leq K} \in L^{2}\left(B_{1}, \operatorname{so}(K) \otimes \mathbb{R}^{2}\right)$. Theorem $\mathrm{C}$ implies that $\phi$ is continuous up to the boundary.

Acknowledgments. The research leading to these results has received funding from the European Research Council under the European Union-Seventh Framework Programme (FP7/2007-2013) / ERC grant agreement no. 267087.

The research of QC was partially supported by NSFC (Grant No. 10871149) and RFDP (Grant No.200804860046). M. Zhu was supported in part by Institute for Mathematical Research (FIM) at ETH Zürich and The Leverhulme Trust. The authors also thank the Max Planck Institute for Mathematics in the Sciences for providing the environment in which this work could be carried out. The authors appreciate the referees' valuable comments and help in improving the contents of this paper.

\section{References}

[1] Albertsson, C., Lindström, U., Zabzine, M.: $N=1$ supersymmetric sigma model with boundaries. I. Comm. Math. Phys. 233, 403-421 (2003) Zbl 1028.81044 MR 1962116

[2] Albertsson, C., Lindström, U., Zabzine, M.: $N=1$ supersymmetric sigma model with boundaries. II. Nuclear Phys. B 678, 295-316 (2004) Zbl 1097.81548 MR 2022994

[3] Atiyah, M. F., Patodi, V. K., Singer, I. M.: Spectral asymmetry and Riemannian geometry, I, II, III. Math. Proc. Cambridge Philos. Soc. 77, 43-69 (1975); 78, 405-432 (1975); 79, 71-99 (1975) Zbl 0314.58016 MR 0397798

[4] Booß-Bavnbek, B., Wojciechowski, K. P.: Elliptic Boundary Problems for the Dirac Operator. Birkhaüser, Basel (1993) Zbl 0797.58004 MR 1233386

[5] Brüning, J., Lesch, M.: Spectral theory of boundary value problems for Dirac type operators. In: Geometric Aspects of Partial Differential Equations (Roskilde, 1998), Contemp. Math. 242, Amer. Math. Soc., 203-215 (1999) Zbl 0945.58026 MR 1714487

[6] Chen, Q., Jost, J., Li, J., Wang, G.: Dirac-harmonic maps. Math. Z. 254, 409-432 (2006) Zbl 1103.53033 MR 2262709 
[7] Chen, Q., Jost, J., Li, J., Wang, G.: Regularity theorems and energy identities for Diracharmonic maps. Math. Z. 251, 61-84 (2005) Zbl 1091.53042 MR 2176464

[8] Deligne, P., et al. (eds.): Quantum Fields and Strings: a Course for Mathematicians, Vol. I. Amer. Math. Soc., Providence, RI, and Inst. Adv. Study, Princeton, NJ (1999) Zbl 0984.00503 MR 1701618

[9] Farinelli, S., Schwarz, G.: On the spectrum of the Dirac operator under boundary conditions. J. Geom. Phys. 28, 67-84 (1998) Zbl 0934.58028 MR 1653130

[10] Gibbons, G. W., Hawking, S. W., Horowitz, G. T., Perry, M. J.: Positive mass theorems for black holes. Comm. Math. Phys. 88, 295-308 (1983) MR 0701918

[11] Giaquinta, M.: Multiple Integrals in the Calculus of Variations and Nonlinear Elliptic Systems. Ann. of Math. Stud. 105, Princeton Univ. Press, Princeton, NJ (1983) Zbl 0516.49003 MR 0717034

[12] Gray, A.: Tubes. 2nd ed., Progr. Math. 221, Birkhäuser, Basel (2004) Z Zbl 1048.53040 MR 2024928

[13] Gulliver, R., Jost, J.: Harmonic maps which solve a free-boundary problem. J. Reine Angew. Math. 381, 61-89 (1987) Zbl 0619.35117 MR 0918841

[14] Hélein, F.: Régularité des applications faiblement harmoniques entre une surface et une variété riemannienne. C. R. Acad. Sci. Paris Sér. I Math. 312, 591-596 (1991) Zbl 0728.35015 MR 1078114

[15] Hélein, F.: Harmonic Maps, Conservation Laws and Moving Frames. 2nd ed., Cambridge Univ. Press (2002) Zbl 1010.58010 MR 1913803

[16] Hijazi, O., Montiel, S., Roldán, A.: Eigenvalue boundary problems for the Dirac operator. Comm. Math. Phys. 231, 375-390 (2002) Zbl 1018.58020 MR 1946443

[17] Hildebrandt, S.: Free boundary problems for minimal surfaces and related questions. In: Frontiers of the Mathematical Sciences: 1985 (New York, 1985), Comm. Pure Appl. Math. 39, no. S, suppl., S111-S138 (1986) Zbl 0601.58021 MR 0861485

[18] Iwaniec, T., Martin, G.: Geometric Function Theory and Non-Linear Analysis. Oxford Math. Monogr., Clarendon Press, New York (2001) Z Zbl 1045.3001 MR 1859913

[19] Jost, J.: Riemannian Geometry and Geometric Analysis. 5th ed., Springer (2008) Zbl 1143.53001 MR 2431897

[20] Jost, J.: Two-Dimensional Geometric Variational Problems, Wiley (1991) Zbl 0729.49001 MR 1100926

[21] Jost, J.: Geometry and Physics. Springer (2009) Zbl 1176.53001 MR 2546999

[22] Müller, F., Schikorra, A.: Boundary regularity via Uhlenbeck-Rivière decomposition. Analysis (München) 29, 199-220 (2009) Zbl 1181.35102 MR 2554638

[23] Raulot, S.: A Sobolev-like inequality for the Dirac operator. J. Funct. Anal. 256, 1588-1617 (2009) Zbl 1166.58017 MR 2490231

[24] Rivière, T.: Conservation laws for conformally invariant variational problems. Invent. Math. 168, 1-22 (2007) Zbl 1128.58010 MR 2285745

[25] Rivière, T., Struwe, M.: Partial regularity for harmonic maps and related problems. Comm. Pure Appl. Math. 61, 451-463 (2008) Zbl 1144.58011 MR 2383929

[26] Scheven, C.: Partial regularity for stationary harmonic maps at a free boundary. Math. Z. 253, 135-157 (2006) Zbl 1092.53050 MR 2206640

[27] Wente, H.: An existence theorem for surfaces of constant mean curvature. J. Math. Anal. Appl. 26, 318-344 (1969) Zbl 0181.11501 MR 0243467

[28] Wang, C., Xu, D.: Regularity of Dirac-harmonic maps. Int. Math. Res. Notices 2009, 37593792 Zbl 1182.58007 MR 2544729

[29] Zhu, M.: Regularity for weakly Dirac-harmonic maps to hypersurfaces. Ann. Global Anal. Geom. 35, 405-412 (2009) Zbl 1171.58005 MR 2506243 